\title{
Risk-averse single machine scheduling: complexity and approximation
}

\author{
Adam Kasperski ${ }^{1} \cdot$ Paweł Zieliński ${ }^{2}$
}

Published online: 28 February 2019

(c) The Author(s) 2019

\begin{abstract}
In this paper, a class of single machine scheduling problems is considered. It is assumed that job processing times and due dates can be uncertain and they are specified in the form of discrete scenario set. A probability distribution in the scenario set is known. In order to choose a schedule, some risk criteria such as the value at risk and conditional value at risk are used. Various positive and negative complexity results are provided for basic single machine scheduling problems. In this paper, new complexity results are shown and some known complexity results are strengthened.
\end{abstract}

Keywords Single machine - Value at risk · Conditional value at risk · Computational complexity · Approximation algorithms . Robust scheduling

\section{Introduction}

Scheduling under risk and uncertainty has attracted considerable attention in the recent literature. In practical applications of scheduling models, the exact values of input parameters, such as job processing times or due dates, are often unknown in advance. Hence, a solution must be computed, before the true realization of the input data is revealed. Typically, a scenario set $\mathcal{U}$ is a part of the input, which contains all possible realizations of the problem parameters, called scenarios. If the probability distribution in $\mathcal{U}$ is unknown, then robust optimization framework can be applied and solution performance in a worst case is optimized. First robust scheduling problems have been discussed in Daniels and Kouvelis (1995), Kouvelis and Yu (1997) and Yu and Kouvelis (1993). Two uncertainty representations, namely discrete and interval ones, were considered. In the former, scenario set $\mathcal{U}$ contains a finite number of distinct scenarios. In the latter, for each uncertain parameter an interval of

Adam Kasperski

adam.kasperski@pwr.edu.pl

Paweł Zieliński

pawel.zielinski@pwr.edu.pl

1 Department of Operations Research, Faculty of Computer Science and Management, Wrocław University of Science and Technology, Wrocław, Poland

2 Department of Computer Science, Faculty of Fundamental Problems of Technology, Wrocław University of Science and Technology, Wrocław, Poland its possible values is specified and $\mathcal{U}$ is the Cartesian product of these intervals. In order to compute a solution, the minmax and minmax regret criteria can be applied. Minmax (regret) scheduling problems have various complexity properties, depending on the cost function and the uncertainty representation [see, e.g., Averbakh (2000), Lebedev and Averbakh (2006), Kasperski (2005), Aissi et al. (2011) and Drwal and Rischke (2016)]. For a survey of minmax (regret) scheduling problems we refer the reader to Kasperski and Zieliński (2014).

The robust scheduling models have well-known drawbacks. Minimizing the maximum cost can lead to very conservative solutions. The reason is that the probability of occurrence of the worst scenario may be very small and the information connected with the remaining scenarios is ignored while computing a solution. One method of overcoming this drawback was given in Kasperski and Zieliński (2016), where the OWA criterion, proposed in Yager (1988), was applied to compute an optimal schedule. In this approach, a set of weights is specified by the decision maker, which reflect his attitude toward a risk. The OWA operator contains the maximum, average and Hurwicz criteria as special cases. However, it does not take into account a probabilistic information, which may be available for scenario set $\mathcal{U}$.

In the case, when a probability distribution in $\mathcal{U}$ is known, the stochastic scheduling models are considered. The parameters of scheduling problem are then random variables with known probability distributions. Under this assumption, the expected solution performance is typically optimized [see, 
e.g., Möhring et al. (1999), Pinedo (2008), Skutella and Uetz (2005) and Skutella et al. (2016)]. However, this criterion assumes that the decision maker is risk neutral and leads to solutions that guarantee an optimal long-run performance. Such a solution may be questionable, for example, if it is implemented only once [see, e.g., Kouvelis and Yu (1997)]. In this case, the decision-maker attitude toward a risk should be taken into account.

In Krokhmal et al. (2002), a criterion called conditional value at risk (CVaR) was applied to a stochastic portfolio selection problem. Using this criterion, the decision maker provides a parameter $\alpha \in[0,1)$, which reflects his attitude toward a risk. When $\alpha=0$, then CVaR becomes the expectation. However, for greater value of $\alpha$, more attention is paid to the worst outcomes, which fits into the robust optimization framework. The conditional value at risk is closely connected with the value at risk (VaR) criterion [see, e.g., Pflug (2000)], which is just the $\alpha$-quantile of a random outcome. Both risk criteria have attracted considerable attention in stochastic optimization [see, e.g., Natarajan et al. (2014), Chang et al. (2017), Nikolova (2010) and Ogryczak (2012)]. This paper is motivated by the recent papers (Sarin et al. 2014; Atakan et al. 2017), in which the following stochastic scheduling models were discussed. We are given a scheduling problem with discrete scenario set $\mathcal{U}$. Each scenario $\xi_{i} \in \mathcal{U}$ is a realization of the problem parameters (for example, processing times and due dates), which can occur with a known positive probability $\operatorname{Pr}\left[\xi_{i}\right]$. The cost of a given schedule is a discrete random variable with the probability distribution induced by the probability distribution in $\mathcal{U}$. The VaR and CVaR criteria, with a fixed level $\alpha$, are used to compute a best solution.

In Sarin et al. (2014) and Atakan et al. (2017), solution methods, based on mixed integer programming models, were proposed to minimize $\mathrm{VaR}$ and $\mathrm{CVaR}$ in scheduling problems with the total weighted tardiness criterion. The aim of this paper is to analyze the models discussed in Sarin et al. (2014) and Atakan et al. (2017) from the complexity point of view. We will consider the class of single machine scheduling problems with basic cost functions, such as the maximum tardiness, the total flow time, the total tardiness and the number of late jobs. We will discuss also the weighted versions of these cost functions. We provide a picture of computational complexity for all these problems by proving some positive and negative complexity results. Since VaR and CVaR generalize the maximum criterion, we can use some results known from robust minmax scheduling. The complexity results for the minmax versions of single machine scheduling problems under discrete scenario set were obtained in Aissi et al. (2011), Aloulou and Croce (2008), Daniels and Kouvelis (1995) and Mastrolilli et al. (2013). In this paper, we will show that some of these results can be strengthened.
This paper is organized as follows. In Sect. 2, we recall the definitions of the VaR and CVaR criteria and show their properties, which will be used later on. In Sect. 3, the problems discussed in this paper are defined. In Sect. 4, some general relationships between the problems with various risk criteria are shown. Finally, Sects. 5 and 6 contain some new negative and positive complexity results for the considered problems. These results are summarized in the tables presented in Sect. 3.

\section{The risk criteria}

Let $Y$ be a random variable. We will consider the following risk criteria (Pflug 2000; Rockafellar and Uryasev 2000):

- Value at risk ( $\alpha$-quantile of $Y$ ):

$$
\operatorname{VaR}_{\alpha}[Y]=\inf \{t: \operatorname{Pr}[Y \leq t] \geq \alpha\}, \alpha \in(0,1]
$$

- Conditional value at risk:

$$
\begin{aligned}
\mathbf{C V a R}_{\alpha}[Y]= & \inf \left\{\gamma+\frac{1}{1-\alpha} \mathbf{E}[Y-\gamma]^{+}: \gamma \in \mathbb{R}\right\}, \\
& \alpha \in[0,1),
\end{aligned}
$$

where $[x]^{+}=\max \{0, x\}$. Assume that $Y$ is a discrete random variable taking nonnegative values $b_{1}, \ldots, b_{K}$. Then, $\operatorname{VaR}_{\alpha}[Y]$ and $\mathbf{C V a R}_{\alpha}[Y]$ can be computed by using the following programs, respectively [see, e.g., Atakan et al. (2017), Ogryczak (2012) and Rockafellar and Uryasev (2000)]:

$$
\begin{array}{lc}
\text { (a) } \min \theta & \text { (b) } \min \gamma+\frac{1}{1-\alpha} \sum_{i \in[K]} \operatorname{Pr}[Y \\
\text { s.t. } b_{k}-\theta \leq M \beta_{k}, \quad k \in[K] & \text { s.t. } \gamma+u_{k} \geq b_{k}, \quad k \in[K] \\
\sum_{k \in[K]} \operatorname{Pr}\left[Y=b_{k}\right] \beta_{k} \leq 1-\alpha & u_{k} \geq 0, \quad k \in[K] \\
\beta_{k} \in\{0,1\}, \quad k \in[K] &
\end{array}
$$

where $M \geq \max \left\{b_{1}, \ldots, b_{K}\right\}$ and $[K]=\{1, \ldots, K\}$. Notice that (1)b is a linear programming problem. In the following, we will use the following dual to (1)b:

$$
\begin{array}{ll}
\max & \sum b_{k \in[K]} b_{k} \\
\text { s.t. } & \sum_{k \in[K]} r_{k}=1 \\
& 0 \leq r_{k} \leq \frac{\operatorname{Pr}\left[Y=b_{k}\right]}{1-\alpha}, \quad k \in[K]
\end{array}
$$

The equality constraint in (2) follows from the fact that $\gamma$ is real-valued decision variable in (1)b. Substituting $r_{k}=$ 


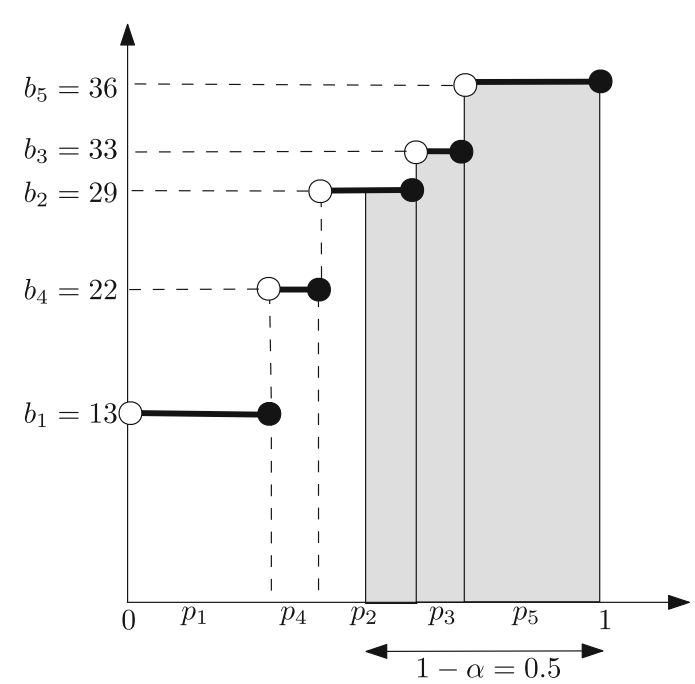

Fig. 1 A computation of $\mathbf{C V a R}_{0.5}[Y]$ for $Y$ taking the values of 13, 29, 33,22 and 36 with the probabilities $0.3,0.2,0.1,0.1,0.3$, respectively, where $\operatorname{Pr}\left[Y=b_{k}\right]=p_{k}$. The value of $\mathbf{C V a R}_{0.5}[Y]$ is the gray area divided by $1-\alpha=0.5$

$q_{k} /(1-\alpha)$ into (2), we get the following equivalent formulation for $\mathbf{C V a R}_{\alpha}[Y]$ :

$$
\begin{aligned}
& \max \frac{1}{1-\alpha} \sum_{k \in[K]} b_{k} q_{k} \\
& \text { s.t. } \quad \sum_{k \in[K]} q_{k}=1-\alpha \\
& 0 \leq q_{k} \leq \operatorname{Pr}\left[Y=b_{k}\right], \quad k \in[K]
\end{aligned}
$$

Program (3) can be solved by using a greedy method, which is illustrated in Fig. 1. Namely, we fix the optimal values of $q_{k}$ by greedily distributing the amount $1-\alpha$ among the largest values of $b_{i}$. It is easy to see that $\mathbf{C V a R}_{0}[Y]=\mathbf{E}[Y]=$ $\sum_{k \in[K]} b_{k} \operatorname{Pr}\left[Y=b_{k}\right]$. On the other hand, $\mathbf{C V a R}_{1-\epsilon}[Y]=$ $\operatorname{VaR}_{1}[Y]=\operatorname{Max}[Y]=\max _{k \in[K]} b_{k}$ for sufficiently small $\epsilon>0$ and any probability distribution.

We now show several properties of the risk measures which will be used later on in this paper.

Lemma 1 Let $Y$ be a discrete random variable which can take $K$ nonnegative values $b_{1}, \ldots, b_{K}$. The following inequalities hold for each $\alpha \in[0,1)$ :

$\mathbf{E}[Y] \leq \mathbf{C V a R}_{\alpha}[Y] \leq \min \left\{\frac{1}{\operatorname{Pr}_{\min }}, \frac{1}{1-\alpha}\right\} \mathbf{E}[Y]$,

where $\operatorname{Pr}_{\min }=\min _{k \in[K]} \operatorname{Pr}\left[Y=b_{k}\right]$.

Proof Fix $\alpha \in[0,1)$. The inequality $\mathbf{E}[Y] \leq \mathbf{C V a R}_{\alpha}[Y]$ follows directly from the definition of the expected value and the conditional value at risk. We now prove the second inequality. Let $r_{1}^{*}, \ldots r_{k}^{*}$ be the optimal values in (2). Then, the inequality

$$
\begin{aligned}
\mathbf{C V a R}_{\alpha}[Y] & =\sum_{k \in[K]} r_{k}^{*} b_{k} \leq \sum_{k \in[K]} \frac{\operatorname{Pr}\left[Y=b_{k}\right]}{(1-\alpha)} b_{k} \\
& =\frac{1}{1-\alpha} \mathbf{E}[Y]
\end{aligned}
$$

holds. Since the value of $\mathbf{C V a R}_{\alpha}[Y]$ is a convex combination of $b_{1}, \ldots, b_{k}$ (see (2)), we have $\mathbf{C V a R}_{\alpha}[Y] \leq \operatorname{Max}[Y]=$ $b_{\max } \leq \sum_{k \in[K]} \frac{\operatorname{Pr}\left[Y=b_{k}\right]}{\operatorname{Pr}_{\min }} b_{k}=\frac{1}{\operatorname{Pr}_{\min }} \mathbf{E}[Y]$, and the lemma follows.

Lemma 2 Let $X$ and $Y$ be two discrete random variables taking nonnegative values $a_{1}, \ldots, a_{K}$, and $b_{1}, \ldots, b_{K}$, respectively, with $\operatorname{Pr}\left[X=a_{i}\right]=\operatorname{Pr}\left[Y=b_{i}\right]$ and $a_{i} \leq$ $\gamma b_{i}$ for each $i \in[K]$ and some fixed $\gamma \geq 0$. Then $\mathbf{C V a R}_{\alpha}[X] \leq \gamma \mathbf{C V a R}_{\alpha}[Y]$ for each $\alpha \in[0,1)$ and $\operatorname{VaR}_{\alpha}[X] \leq \gamma \operatorname{VaR}_{\alpha}[Y]$ for each $\alpha \in(0,1]$.

Proof Let us compute $\mathbf{C V a R}_{\alpha}[X]$ by using (2) and denote by $r_{k}^{*}, k \in[K]$, the optimal values in (2). Then, $\mathbf{C V a R}_{\alpha}[X]=$ $\sum_{k \in[K]} r_{k}^{*} a_{k} \leq \gamma \sum_{k \in[K]} r_{k}^{*} b_{k} \leq \gamma \mathbf{C V a R}_{\alpha}[Y]$. Let us compute $\mathbf{V a R}_{\alpha}[Y]$ by solving the problem (1)a. Let $\theta^{*}, \beta_{k}^{*}$, $k \in[K]$, be an optimal solution to (1)a. Since $\gamma \geq 0$, the constraint $\gamma b_{k}-\gamma \theta^{*} \leq \gamma M \beta_{k}^{*}$ holds for each $k \in[K]$. By $a_{k} \leq \gamma b_{k}$ for each $k \in[K]$, we get $a_{k}-\gamma \theta^{*} \leq M^{\prime} \beta_{k}^{*}$, where $M^{\prime}=\gamma M \geq \max \left\{a_{1}, \ldots, a_{K}\right\}, k \in[K]$. In consequence,

$$
\begin{aligned}
& a_{k}-\gamma \theta^{*} \leq M^{\prime} \beta_{k}^{*} \quad k \in[K] \\
& \sum_{k \in[K]} \operatorname{Pr}\left[X=a_{k}\right] \cdot \beta_{k}^{*} \leq 1-\alpha
\end{aligned}
$$

and $\mathbf{V a R}_{\alpha}[X] \leq \gamma \theta^{*}=\gamma \operatorname{VaR}_{\alpha}[Y]$.

\section{Problem formulations}

We are given a set $J$ of $n$ jobs, which can be partially ordered by some precedence constraints. Namely, $i \rightarrow j$ means that job $j$ cannot start before job $i$ is completed. For each job $j \in J$, a nonnegative processing time $p_{j}$, a nonnegative due date $d_{j}$ and a nonnegative weight $w_{j}$ can be specified. A schedule $\pi$ is a feasible (i.e., preserving the precedence constraints) permutation of the jobs and $\Pi$ is the set of all feasible schedules. We will use $C_{j}(\pi)$ to denote the completion time of job $j$ in schedule $\pi$. Obeying the standard notation, we will use $T_{j}(\pi)=\left[C_{j}(\pi)-d_{j}\right]^{+}$to define the tardiness of $j$ in $\pi$, and $U_{j}(\pi)=1$ if $C_{j}(\pi)>d_{j}$ (job $j$ is late in $\pi$ ) and $U_{j}(\pi)=0$ (job $j$ is on-time in $\pi$ ), otherwise. In the deterministic case, we seek a schedule $\pi \in \Pi$ that minimizes a given cost function $f(\pi)$. The basic cost functions are the total flow time $\sum_{j \in J} C_{j}(\pi)$, the total tardiness $\sum_{j \in J} T_{j}(\pi)$, the maximum tardiness $\max _{j \in J} T_{j}(\pi)$ and the total number of late jobs $\sum_{j \in J} U_{j}(\pi)$. We can also consider the weighted versions of these functions. Scheduling problems $\mathcal{P}$ will be 
Fig. 2 A sample scheduling problem $1 \| \sum C_{j}$ with 5 processing time scenarios

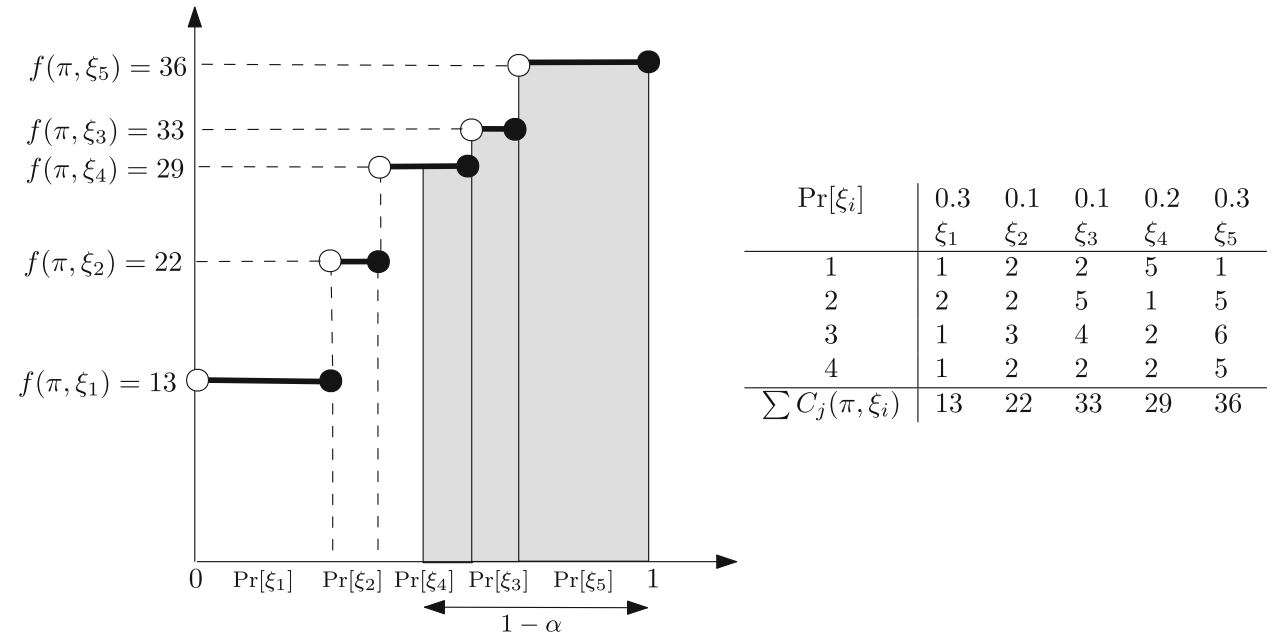

denoted by means of the standard Graham's notation [see, e.g., Brucker (2007)].

In this paper, we assume that job processing times and due dates can be uncertain. The uncertainty is modeled by a discrete scenario set $\mathcal{U}=\left\{\xi_{1}, \xi_{2}, \ldots, \xi_{K}\right\}$. Each realization of the parameters $\xi \in \mathcal{U}$ is called a scenario. For each scenario $\xi \in \mathcal{U}$, a probability $\operatorname{Pr}[\xi]$ of its occurrence is known. Without loss of generality, we can assume $\operatorname{Pr}[\xi]>0$. We will use $p_{j}(\xi)$ and $d_{j}(\xi)$ to denote the processing time and due date of job $j$ under scenario $\xi \in \mathcal{U}$, respectively. We will denote by $C_{j}(\pi, \xi), T_{j}(\pi, \xi)$ and $U_{j}(\pi, \xi)$ the completion time, tardiness and unit penalty of job $\pi$, respectively, under scenario $\xi \in \mathcal{U}$. Also, $f(\pi, \xi)$ stands for the cost of schedule $\pi$ under scenario $\xi \in \mathcal{U}$. Given a feasible schedule $\pi \in \Pi$, we denote by $F(\pi)$ a random cost of $\pi$. Notice that $F(\pi)$ is a discrete random variable with the probability distribution induced by the probability distribution in $\mathcal{U}$.

For a fixed value of $\alpha$, we can compute a performance measure of $\pi$, namely the expected cost $\mathbf{E}[\mathrm{F}(\pi)]$, the maximum cost $\operatorname{Max}[\mathrm{F}(\pi)]$, the value at risk $\operatorname{VaR}_{\alpha}[\mathrm{F}(\pi)]$ and the conditional value at risk $\mathbf{C V a R} \mathbf{R}_{\alpha}[\mathrm{F}(\pi)]$. A sample problem $1 \| \sum C_{j}$ with 4 jobs and 5 processing time scenarios is shown in Fig. 2. Let $\pi=(1,2,3,4)$. It is easily seen that $\mathbf{E}[\mathrm{F}(\pi)]=26, \operatorname{VaR}_{0.5}[\mathrm{~F}(\pi)]=29, \mathbf{C V a R}_{0.5}[\mathrm{~F}(\pi)]=34$ and $\operatorname{Max}[\mathrm{F}(\pi)]=36$.

In this paper, we will study the problems $\mathrm{MIN}-\mathrm{VAR}_{\alpha} \mathcal{P}$, MIN-CVAR $\alpha \mathcal{P}$, MIN-EXP $\mathcal{P}$, and MIN-MAX $\mathcal{P}$, in which we minimize the corresponding performance measure for a fixed $\alpha$ and a specific single machine scheduling problem $\mathcal{P}$, under a given scenario set $\mathcal{U}$. Notice that the robust MIN-MAX $\mathcal{P}$ problem is a special case of both $\mathrm{MIN}-\mathrm{VAR}_{\alpha} \mathcal{P}$ and MIN-CVAR $\alpha \mathcal{P}$. Also, MIN-EXP $\mathcal{P}$ is a special case of MIN-CVAR ${ }_{\alpha} \mathcal{P}$.

In the next sections, we provide a number of new positive and negative complexity and approximation results for basic single machine scheduling problems $\mathcal{P}$. Tables 1,2 and 3 summarize the known and new results. In Table 1, the negative results for uncertain due dates and deterministic processing times are shown. In Table 2, the negative results for uncertain processing times and deterministic due dates are presented. Finally, in Table 3, some positive results are shown.

\section{Some general properties}

In this section, we will show some general relationships between the problems with various performance criteria. These properties will be used later to establish some positive and negative complexity results for particular problems.

Theorem 1 The following statements hold:

1. If $\mathrm{MIN}-\mathrm{EXP} \mathcal{P}$ is approximable within $\sigma>1$ (for $\sigma=1$ it is polynomially solvable), then $\mathrm{MIN}-\mathrm{CVAR}_{\alpha} \mathcal{P}$ is approximable within $\sigma \rho$, where $\rho=\min \left\{\frac{1}{\operatorname{Pr}_{\min }}, \frac{1}{1-\alpha}\right\}$, for each constant $\alpha \in[0,1)$.

2.a If MIN-EXP $\mathcal{P}$ with $K$-scenarios is (strongly) NP-hard, then $\mathrm{MIN}-\mathrm{CVAR}_{\alpha} \mathcal{P}$ with $K+1$ scenarios is also (strongly) NP-hard for each constant $\alpha \in[0,1)$.

2.b If MIN-EXP $\mathcal{P}$ with $K$-scenarios is hard to approximate within $\rho>1$, then $\mathrm{MIN}-\mathrm{CVAR}_{\alpha} \mathcal{P}$ with $K+1$ scenarios is also hard to approximate within $\rho>1$ for each constant $\alpha \in[0,1)$.

Proof We first prove assertion 1. Let $\pi^{*}$ minimize the expected cost and $\pi^{\prime}$ minimize the conditional value at risk for a fixed $\alpha \in[0,1)$. We will denote by $\hat{\pi}$ a $\sigma$-approximation schedule for MIN-EXP $\mathcal{P}$. Using Lemma 1 we get

$$
\begin{aligned}
\operatorname{CVaR}_{\alpha}[\mathrm{F}(\hat{\pi})] & \leq \rho \mathbf{E}[\mathrm{F}(\hat{\pi})] \leq \sigma \rho \mathbf{E}\left[\mathrm{F}\left(\pi^{*}\right)\right] \\
& \leq \sigma \rho \mathbf{E}\left[\mathrm{F}\left(\pi^{\prime}\right)\right] \leq \sigma \rho \mathbf{C V a R} \mathbf{R}_{\alpha}\left[\mathrm{F}\left(\pi^{\prime}\right)\right]
\end{aligned}
$$

and the assertion follows. 
Table 1 Complexity results for uncertain due dates (processing times are deterministic)

\begin{tabular}{|c|c|c|c|c|}
\hline $\mathcal{P}$ & $\operatorname{MIN}-\operatorname{EXP} \mathcal{P}$ & $\mathrm{MIN}-\mathrm{VAR}_{\alpha} \mathcal{P}$ & $\mathrm{MIN}-\mathrm{CVAR}_{\alpha} \mathcal{P}$ & MIN-MAX $\mathcal{P}$ \\
\hline $1\left|p_{j}=1\right| \max T_{j}$ & $\begin{array}{l}\text { Str. NP-hard not appr. within } \\
\frac{7}{6}-\epsilon, \epsilon>0 \text { (Kasperski and } \\
\text { Zieliński 2016) }\end{array}$ & $\begin{array}{l}\text { Str. NP-hard not at all appr. } \\
\text { for any } \alpha \in(0,1)\end{array}$ & $\begin{array}{l}\text { Str. NP-hard not appr. } \\
\text { within } \frac{7}{6}-\epsilon, \epsilon>0 \\
\text { for any } \alpha \in[0,1)\end{array}$ & $\begin{array}{l}\text { Poly. sol. (Kasperski } \\
\text { and Zieliński 2016) }\end{array}$ \\
\hline $1\left|p_{j}=1\right| \sum U_{j}$ & Poly sol. (assignment) & $\begin{array}{l}\text { Str. NP-hard not at all appr. } \\
\text { for any } \alpha \in(0,1)\end{array}$ & $\begin{array}{l}\text { Str. NP-hard for any } \\
\quad \alpha \in(0,1)\end{array}$ & $\begin{array}{l}\text { Str. NP-hard not appr. } \\
\text { for any constant } \gamma>1\end{array}$ \\
\hline $1 \| \sum U_{j}$ & NP-hard & As above & As above & As above \\
\hline $1\left|p_{j}=1\right| \sum T_{j}$ & Poly sol. (assignment) & $\begin{array}{l}\text { Str. NP-hard not at all appr. } \\
\text { for any } \alpha \in(0,1)\end{array}$ & $\begin{array}{l}\text { Str. NP-hard for any } \\
\qquad \alpha \in(0,1)\end{array}$ & $\begin{array}{l}\text { Str. NP-hard not appr. } \\
\text { within } \frac{5}{4}-\epsilon, \epsilon>0\end{array}$ \\
\hline $1 \| \sum T_{j}$ & Str. NP-hard & As above & As above & As above \\
\hline
\end{tabular}

Table 2 Complexity results for uncertain processing times (the due dates are deterministic)

\begin{tabular}{|c|c|c|c|c|}
\hline $\mathcal{P}$ & MIN-EXP $\mathcal{P}$ & $\mathrm{MIN}-\mathrm{VAR}_{\alpha} \mathcal{P}$ & $\mathrm{MIN}-\mathrm{CVAR}_{\alpha} \mathcal{P}$ & MIN-MAX $\mathcal{P}$ \\
\hline $1 \| \sum C_{j}$ & Poly sol. & $\begin{array}{l}\text { Str. NP-hard not appr. } \\
\text { within } \frac{6}{5}-\epsilon, \epsilon>0\end{array}$ & $\begin{array}{r}\text { Str. NP-hard for } \\
\text { any } \alpha \in(0,1)\end{array}$ & $\begin{array}{l}\text { Str. NP-hard not appr. within } \frac{6}{5}-\epsilon, \\
\epsilon>0 \text { (Kouvelis and Yu 1997; } \\
\text { Mastrolilli et al. 2013) }\end{array}$ \\
\hline $1 \| \sum U_{j}$ & Open & $\begin{array}{l}\text { Str. NP-hard for any } \\
\quad \alpha \in(0,1]\end{array}$ & $\begin{array}{r}\text { Str. NP-hard for } \\
\text { any } \alpha \in(0,1)\end{array}$ & Str. NP-hard \\
\hline $1 \| \sum T_{j}$ & NP-hard (Lawler 1977) & $\begin{array}{l}\text { Str. NP-hard not appr. } \\
\text { within } \frac{6}{5}-\epsilon, \epsilon>0\end{array}$ & $\begin{array}{l}\text { Str. NP-hard for } \\
\text { any } \alpha \in(0,1)\end{array}$ & $\begin{array}{l}\text { Str. NP-hard not appr. within } \frac{6}{5}-\epsilon \text {, } \\
\qquad \begin{array}{l}\epsilon>0\end{array}\end{array}$ \\
\hline
\end{tabular}

Table 3 Positive complexity results

\begin{tabular}{|c|c|c|c|c|}
\hline $\mathcal{P}$ & MIN-EXP $\mathcal{P}$ & $\mathrm{MIN}-\mathrm{VAR}_{\alpha} \mathcal{P}$ & $\mathrm{MIN}-\mathrm{CVAR}_{\alpha} \mathcal{P}$ & MIN-MAX $\mathcal{P}$ \\
\hline $1 \mid$ prec $\mid \max w_{j} T_{j}$ with $\tilde{d}_{j}, \tilde{p}_{j}, w_{j}$ & $\begin{array}{l}O\left(f_{\max }^{K} K n^{2}\right) \text { FPTAS } \\
\text { for const. } K\end{array}$ & $\begin{array}{l}O\left(f_{\max }^{K} K n^{2}\right) \text { FPTAS } \\
\text { for const. } K\end{array}$ & $\begin{array}{l}O\left(f_{\max }^{K} K n^{2}\right) \\
\text { FPTAS for const. } \\
K\end{array}$ & $\begin{array}{l}O\left(K n^{2}\right)(\text { Kasperski and } \\
\text { Zieliński 2016) }\end{array}$ \\
\hline $1 \mid$ prec $\mid \sum w_{j} C_{j}$ with $\tilde{p}_{j}, w_{j}$ & As the determ. problem & $\begin{array}{l}\text { Appr. within } 2 \text { for const. } \\
K\end{array}$ & Appr. within 2 & $\begin{array}{l}\text { Appr. within } 2 \text { (Mastrolilli } \\
\text { et al. 2013) }\end{array}$ \\
\hline $1\left|\operatorname{prec}^{*}\right| \sum w_{j} C_{j}$ with $\tilde{p}_{j}, w_{j}$ & Poly sol. & $\begin{array}{l}\text { Appr. within } 2 \text { for const. } \\
K\end{array}$ & $\begin{array}{l}\text { Appr. within } \\
\min \left\{\frac{1}{1-\alpha}, 2\right\}\end{array}$ & $\begin{array}{l}\text { Appr. within } 2 \text { (Mastrolilli } \\
\text { et al. 2013) }\end{array}$ \\
\hline $1\left|p_{j}=1\right| \sum w_{j} U_{j}$ with $\tilde{d}_{j}, w_{j}$ & Poly sol. & - & $\begin{array}{l}\text { Appr. within } \\
\qquad \min \left\{\frac{1}{\operatorname{Pr}_{\min }}, \frac{1}{1-\alpha}\right\}\end{array}$ & Appr. within $K$ \\
\hline $1 \| \sum w_{j} U_{j}$ with $\tilde{d}_{j}, p_{j}, w_{j}$ & $\begin{array}{l}\text { Appr. within } 4+\epsilon \text {, } \\
\qquad \epsilon>0\end{array}$ & - & $\begin{array}{l}\text { Appr. within } \\
\quad \min \left\{\frac{4+\epsilon}{\operatorname{Pr}_{\min }}, \frac{4+\epsilon}{1-\alpha}\right\}\end{array}$ & $\begin{array}{l}\text { Appr. within }(4+\epsilon) K \\
\quad \epsilon>0\end{array}$ \\
\hline $1\left|p_{j}=1\right| \sum w_{j} T_{j}$ with $\tilde{d}_{j}, w_{j}$ & Poly sol. & - & $\begin{array}{l}\text { Appr. within } \\
\qquad \min \left\{\frac{1}{\operatorname{Pr}_{\min }}, \frac{1}{1-\alpha}\right\}\end{array}$ & Appr. within $K$ \\
\hline $1 \| \sum w_{j} T_{j}$ with $\tilde{d}_{j}, p_{j}, w_{j}$ & $\begin{array}{l}\text { Appr. within } 4+\epsilon \text {, } \\
\qquad>0\end{array}$ & - & $\begin{array}{l}\text { Appr. within } \\
\qquad \min \left\{\frac{4+\epsilon}{\operatorname{Pr}_{\min }}, \frac{4+\epsilon}{1-\alpha}\right\}\end{array}$ & $\begin{array}{l}\text { Appr. within }(4+\epsilon) K \\
\quad \epsilon>0\end{array}$ \\
\hline
\end{tabular}

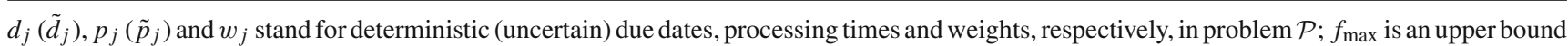
on the cost of any schedule under any scenario; $\operatorname{prec}^{*}$ is a polynomially solvable structure of the precedence constraints; $\operatorname{Pr}_{\min }=\min _{k \in[K]} \operatorname{Pr}\left[\xi_{k}\right]$

In order to prove assertion 2 , consider an instance of MIN-EXP $\mathcal{P}$ with $\mathcal{U}=\left\{\xi_{1}, \ldots, \xi_{K}\right\}$. Fix $\alpha \in(0,1)$ (the statement trivially holds for $\alpha=0$ ) and add one additional scenario $\xi^{\prime}$ under which the cost of each schedule is 0. (For example, all job processing times are 0 under $\xi^{\prime}$.) We fix $\operatorname{Pr}^{\prime}\left[\xi^{\prime}\right]=\alpha$ and $\operatorname{Pr}^{\prime}\left[\xi_{i}\right]=\operatorname{Pr}\left[\xi_{i}\right] \cdot(1-\alpha)$ for each $i \in[K]$. Denote by $\mathrm{F}^{\prime}(\pi)$ the random cost of $\pi$ under the new scenario set $\mathcal{U}^{\prime}$. For each schedule $\pi$, we get (see Fig. 3):

$$
\begin{aligned}
\operatorname{CVaR}_{\alpha}\left[\mathrm{F}^{\prime}(\pi)\right] & =\frac{1}{1-\alpha} \sum_{i \in[K]} \operatorname{Pr}^{\prime}\left[\xi_{i}\right] f\left(\pi, \xi_{i}\right) \\
& =\sum_{i \in[K]} \operatorname{Pr}\left[\xi_{i}\right] f\left(\pi, \xi_{i}\right)=\mathbf{E}[\mathrm{F}(\pi)] .
\end{aligned}
$$

Hence, there is a cost preserving reduction from MIN-EXP $\mathcal{P}$ with $K$ scenarios to MIN-CVAR $\mathcal{P}$ with $K+1$ scenarios and the theorem follows. 


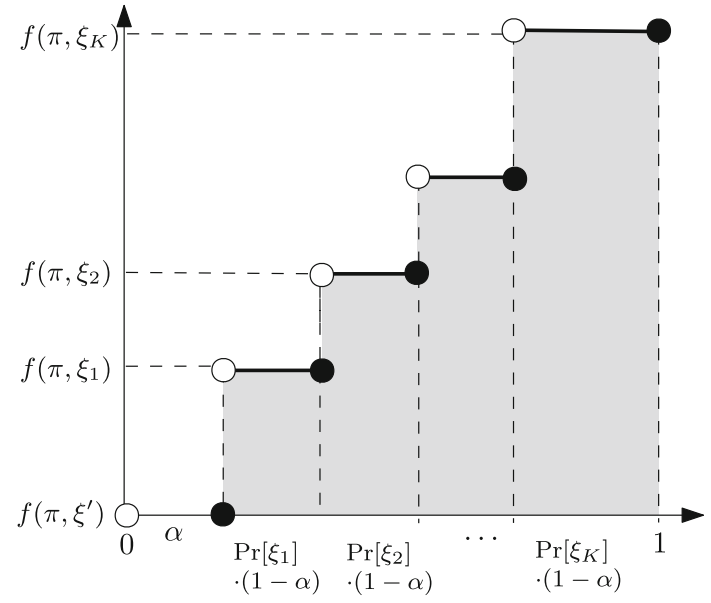

Fig. 3 Illustration of the proof of Theorem 1

Theorem 2 Assume that $w_{j}=1$ for each job $j \in J$ in problem $\mathcal{P}$. The following statements hold:

1.a If MIN-MAX $\mathcal{P}$ with $K \geq 2$ scenarios is (strongly) $N P$-hard, then MIN-VAR $\mathrm{V}_{\alpha} \mathcal{P}$ with $K+1$ scenarios is (strongly) NP-hard for each constant $\alpha \in(0,1]$.

1.b If MIN-MAX $\mathcal{P}$ with $K \geq 2$ scenarios is hard to approximate within $\rho>1$, then $\mathrm{MIN}-\mathrm{VAR}_{\alpha} \mathcal{P}$ with $K+1$ scenarios is hard to approximate within $\rho>1$ for each constant $\alpha \in(0,1]$.

2. If MIN-MAX $\mathcal{P}$ with $K \geq 2$ scenarios is (strongly) $N P$ hard, then MIN-CVAR $\mathrm{R}_{\alpha} \mathcal{P}$ with $K+1$ scenarios is (strongly) NP-hard for each constant $\alpha \in(0,1)$

Proof Choose an instance of the MIN-MAX $\mathcal{P}$ problem with $\mathcal{U}=\left\{\xi_{1}, \ldots, \xi_{K}\right\}, K \geq 2$. Fix $\alpha \in(0,1)$ and create $\mathcal{U}^{\prime}$ by adding to $\mathcal{U}$ a dummy scenario $\xi^{\prime}$ such that the cost of each schedule under $\xi^{\prime}$ equals $M$ and $M \geq f\left(\pi, \xi_{i}\right)$ for each $i \in[K]$ and each $\pi \in \Pi$. It is enough to fix $p_{j}\left(\xi^{\prime}\right)=$ $p_{\max }$ and $d_{j}\left(\xi^{\prime}\right)=d_{\min }$ for each job $j \in J$, where $p_{\max }=$ $\max _{j \in J, i \in[K]} p_{j}\left(\xi_{i}\right)$ is the maximum job processing time and $d_{\text {min }}=\min _{j \in J, i \in[K]} d_{j}\left(\xi_{i}\right)$ is the minimum due date over all scenarios. For each of the two assertions, we define an appropriate probability distribution in $\mathcal{U}^{\prime}$. We will use $\mathrm{F}^{\prime}(\pi)$ to denote the random cost of $\pi$ under $\mathcal{U}^{\prime}$.

In order to prove statement 1 , we fix $\operatorname{Pr}\left[\xi^{\prime}\right]=1-\alpha$ and $\operatorname{Pr}\left[\xi_{i}\right]=\frac{\alpha}{K}$ for each $i \in[K]$ (see Fig. 4a). The equality $\operatorname{VaR}_{\alpha}\left[\mathrm{F}^{\prime}(\pi)\right]=\operatorname{Max}[\mathrm{F}(\pi)]$ holds. Hence, there is a cost preserving reduction from MIN-MAX $\mathcal{P}$ with $K$ scenarios to MIN- $\mathrm{VAR}_{\alpha} \mathcal{P}$ with $K+1$ scenarios and the statement follows. Note that statement 1 holds trivially for $\alpha=1$.

Let us now prove statement 2 . Assume first that $1-\alpha<\frac{1}{K}$. Define $\operatorname{Pr}\left[\xi_{i}\right]=\frac{1}{K}$ for each $i \in[K]$ and $\operatorname{Pr}\left[\xi^{\prime}\right]=0$. The dummy scenario is not used in this case. We get $\operatorname{CVaR}_{\alpha}\left[\mathrm{F}^{\prime}(\pi)\right]=\operatorname{Max}[\mathrm{F}(\pi)]$ and the statement is true. Consider the case, when $1-\alpha \geq \frac{1}{K}$. Fix $\operatorname{Pr}\left[\xi^{\prime}\right]=\gamma$ and
$\operatorname{Pr}\left[\xi_{i}\right]=\beta$ for each $i \in[K]$, where $\gamma$ and $\beta$ satisfy the following system of equations (see Fig. 4b):

$$
\left\{\begin{array}{l}
\beta+\gamma=1-\alpha \\
K \beta+\gamma=1
\end{array}\right.
$$

In consequence, $\beta=\frac{\alpha}{K-1}$ and $\gamma=1-\frac{K \alpha}{K-1}$. Observe that $\beta>1$ as $\alpha \in(0,1)$ and $K \geq 2$, and $\gamma \geq 0$, because $\frac{K \alpha}{K-1} \leq 1$.

For each schedule $\pi$, we get

$\operatorname{CVaR}_{\alpha}\left[\mathrm{F}^{\prime}(\pi)\right]=\frac{1}{1-\alpha}(\beta \cdot \operatorname{Max}[\mathrm{F}(\pi)]+\gamma M)$.

Hence, MIN-MAX $\mathcal{P}$ and the corresponding instance of MIN-CVAR $\alpha \mathcal{P}$ have the same optimal solutions and the theorem follows.

\section{Negative complexity results}

In this section, we will prove some negative complexity results for basic single machine scheduling problems. These results are summarized in Tables 1 and 2 .

\subsection{Uncertain due dates}

We first address the problem of minimizing the value at risk criterion. The following theorem characterizes the complexity of some basic problems:

Theorem 3 For each $\alpha \in(0,1)$, MIN-VAR $\mathrm{V}_{\alpha} \mathcal{P}$ is strongly $N P$-hard and not at all approximable, when $\mathcal{P} \in\left\{1 \mid p_{j}=\right.$ $\left.1\left|\max T_{j}, 1\right| p_{j}=1\left|\sum T_{j}, 1\right| p_{j}=1 \mid \sum U_{j}\right\}$ and only due dates are uncertain.

Proof Consider an instance of the following NP-hard MiN 3- SAT problem (Kohli et al. 1994; Avidor and Zwick 2002). We are given boolean variables $x_{1}, \ldots, x_{n}$, a collection of clauses $\mathcal{C}_{1}, \ldots \mathcal{C}_{m}$, where each clause is a disjunction of at most 3 literals (variables or their negations), and we ask if there is an assignment to the variables which satisfies at most $L<m$ clauses. We can ask equivalently, if there is an assignment to the variables for which at least $l=m-L$ clauses are not satisfied.

Given an instance of MiN 3- SAT, we create two jobs $J_{x_{i}}$ and $J_{\bar{x}_{i}}$ for each variable $x_{i}, i \in[n]$. A due date scenario $\xi_{i}$ corresponds to clause $\mathcal{C}_{i}=\left(l_{1} \vee l_{2} \vee l_{3}\right)$ and is formed as follows. For each $q=1,2,3$, if $l_{q}=x_{j}$, then the due date of $J_{x_{j}}$ is $2 j-1$ and the due date of $J_{\bar{x}_{j}}$ is $2 j$; if $l_{q}=\bar{x}_{j}$, then the due date of $J_{x_{j}}$ is $2 j$ and the due date of $J_{\bar{x}_{j}}$ is $2 j-1$; if neither $x_{j}$ nor $\bar{x}_{j}$ appears in $\mathcal{C}_{i}$, then the due dates of $J_{x_{j}}$ and $J_{\bar{x}_{j}}$ are set to $2 j$. An example is shown in Table 4. 

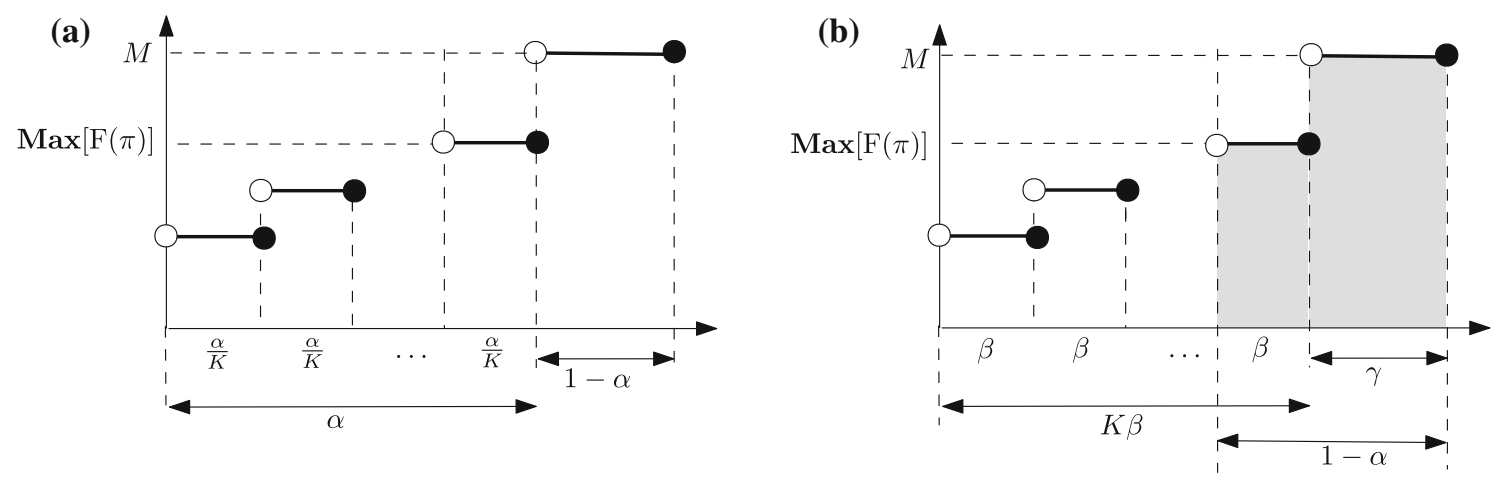

Fig. 4 Illustration of the proof of Theorem 2

Table 4 The set of jobs and the due date scenarios for the formula $\left(x_{1} \vee \bar{x}_{2} \vee \bar{x}_{3}\right) \wedge\left(\bar{x}_{2} \vee\right.$ $\left.\bar{x}_{3} \vee x_{4}\right) \wedge\left(\bar{x}_{1} \vee x_{2} \vee \bar{x}_{4}\right) \wedge$ $\left(x_{1} \vee x_{2} \vee x_{3}\right) \wedge\left(x_{1} \vee x_{3} \vee \bar{x}_{4}\right)$

\begin{tabular}{llllll}
\hline & $\xi_{1}$ & $\xi_{2}$ & $\xi_{3}$ & $\xi_{4}$ & $\xi_{5}$ \\
\hline$J_{x_{1}}$ & 1 & 2 & 2 & 1 & 1 \\
$J_{\bar{x}_{1}}$ & 2 & 2 & 1 & 2 & 2 \\
$J_{x_{2}}$ & 4 & 4 & 3 & 3 & 4 \\
$J_{\bar{x}_{2}}$ & 3 & 3 & 4 & 4 & 4 \\
$J_{x_{3}}$ & 6 & 6 & 6 & 5 & 5 \\
$J_{\bar{x}_{3}}$ & 5 & 5 & 6 & 6 & 6 \\
$J_{x_{4}}$ & 8 & 7 & 8 & 8 & 8 \\
$J_{\bar{x}_{4}}$ & 8 & 8 & 7 & 8 & 7 \\
\hline
\end{tabular}

Let us define a subset of the schedules $\Pi^{\prime} \subseteq \Pi$ such that each schedule $\pi \in \Pi^{\prime}$ is of the form $\pi=$ $\left(J_{1}, J_{1}^{\prime}, J_{2}, J_{2}^{\prime}, \ldots, J_{n}, J_{n}^{\prime}\right)$, where $J_{j}, J_{j}^{\prime} \in\left\{J_{x_{j}}, J_{\bar{x}_{j}}\right\}$ for $j \in[n]$. Observe that $\Pi^{\prime}$ contains exactly $2^{n}$ schedules and each such a schedule corresponds to the assignment to the variables such that $x_{j}=0$ if $J_{x_{j}}$ is processed before $J_{\bar{x}_{j}}$ and $x_{j}=1$ otherwise. Note that this correspondence is one-to-one. In the following, we assume that $f\left(\pi, \xi_{i}\right)$ is the maximum tardiness, or the total tardiness, or the sum of unit penalties in $\pi$ under $\xi_{i}$. The reasoning will be the same for each of these cost functions. If $\pi \notin \Pi^{\prime}$, then $f\left(\pi, \xi_{i}\right)>0$ for each scenario $\xi_{i}$. Indeed, suppose that $\pi \notin \Pi^{\prime}$ and let $J_{j}\left(J_{j}^{\prime}\right)$ be the last job in $\pi$ which is not placed properly, i.e., $J_{j},\left(J_{j}^{\prime}\right) \notin\left\{J_{x_{j}}, J_{\bar{x}_{j}}\right\}$. Then, $J_{j}\left(J_{j}^{\prime}\right)$ is late under all scenarios. On the other hand, if $\pi \in \Pi^{\prime}$, then the number of scenarios under which no job is late is equal to the number of unsatisfiable clauses for the assignment corresponding to $\pi$. Fix $\alpha \in(0,1)$. We will add to $\mathcal{U}$ one additional scenario $\xi^{\prime}$ and define a probability distribution in $\mathcal{U}$, depending on the fixed $\alpha$, so that the answer to MIN 3- SAT is yes if and only if there is schedule $\pi$ for which $\operatorname{VaR}_{\alpha}[\mathrm{F}(\pi)] \leq 0$. This will prove the stated result. We consider two cases:

1. $l / m \geq \alpha$. We create dummy scenario $\xi^{\prime}$ under which the due date of all jobs is equal to 0 . The probability of this scenario is equal to $\frac{l-\alpha m}{l}$. The probability of each of the remaining scenarios is equal to $\frac{1}{m}\left(1-\frac{l-\alpha m}{l}\right)=$ $\frac{\alpha}{l}$. Assume that the answer to Min 3-SAT is yes. So, there is an assignment to the variables which satisfies at most $m-l$ clauses. By the above construction, there is a schedule $\pi \in \Pi^{\prime}$ whose cost is positive under at most $m-l$ scenarios plus the dummy one. It holds

$\operatorname{Pr}[\mathrm{F}(\pi)>0] \leq \frac{l-\alpha m}{l}+(m-l) \frac{\alpha}{l}=1-\alpha$.

Hence, $\operatorname{Pr}[\mathrm{F}(\pi) \leq 0] \geq \alpha$ and $\operatorname{VaR}_{\alpha}[\mathrm{F}(\pi)] \leq 0$. Assume that the answer to MIN 3- SAT is no. Then, for every schedule $\pi$ there are more than $m-l$ scenarios under which the cost of $\pi$ is positive plus the dummy one. Hence, $\operatorname{Pr}[\mathrm{F}(\pi)>0]>(1-\alpha)$ and $\operatorname{Pr}[\mathrm{F}(\pi) \leq 0]<\alpha$. In consequence, $\operatorname{VaR}_{\alpha}[\mathrm{F}(\pi)]>0$.

2. $l / m<\alpha$. We create dummy scenario $\xi^{\prime}$ under which the due date of each job equals $2 n$. The probability of the dummy scenario is $\frac{m \alpha-l}{m-l}$. The probability of each of the remaining scenarios is equal to $\frac{1}{m}\left(1-\frac{m \alpha-l}{m-l}\right)=\frac{1-\alpha}{m-l}$. Assume that the answer to Min 3- SAT is yes. So, there is an assignment to the variables which satisfies at most $m-l$ clauses. By the construction, there is a schedule $\pi$ whose cost is positive under at most $m-l$ scenarios. Hence,

$$
\begin{aligned}
\operatorname{Pr}[\mathrm{F}(\pi) & \leq 0]=1-\operatorname{Pr}[\mathrm{F}(\pi)>0] \\
& \geq 1-(m-l) \frac{1-\alpha}{m-l}=\alpha
\end{aligned}
$$

and $\mathbf{V a R}_{\alpha}[\mathrm{F}(\pi)] \leq 0$. Assume that the answer to MIN 3- SAT is no. Then, for each assignment more than $m-l$ clauses are satisfied. By the construction, for every schedule $\pi$ there are more than $m-l$ scenarios under which the cost $\pi$ is positive. Therefore, $\operatorname{Pr}[\mathrm{F}(\pi)>0]>$ $(m-l) \frac{1-\alpha}{m-l}=(1-\alpha)$ and $\operatorname{Pr}[\mathrm{F}(\pi) \leq 0]<\alpha$, so $\mathbf{V a R}_{\alpha}[\mathrm{F}(\pi)]>0$.

It follows from Theorem 3 that the problem MIN- $\mathrm{VAR}_{\alpha} 1$ $\| \sum w_{j} T_{j}$, discussed in Atakan et al. (2017), is strongly 
Table 5 The set of jobs and the due date scenarios for the formula $\left(x_{1} \vee\right.$ $\left.\bar{x}_{2} \vee \bar{x}_{3}\right) \wedge\left(\bar{x}_{2} \vee \bar{x}_{3} \vee x_{4}\right) \wedge\left(\bar{x}_{1} \vee x_{2} \vee \bar{x}_{4}\right) \wedge\left(x_{1} \vee x_{2} \vee x_{3}\right) \wedge\left(x_{1} \vee x_{3} \vee \bar{x}_{4}\right)$. Schedule $\left.\pi=\left(J_{x_{1}}, J_{\bar{x}_{1}}, J_{\bar{x}_{2}}, J_{x_{2}}, J_{x_{3}}, J_{\bar{x}_{3}}, J_{\bar{x}_{4}}, J_{x_{4}}\right)\right)$ corresponds to a truth assignment

\begin{tabular}{llllllllll}
\hline & $\xi_{1}$ & $\xi_{2}$ & $\xi_{3}$ & $\xi_{4}$ & $\xi_{5}$ & $\xi_{1}^{\prime}$ & $\xi_{2}^{\prime}$ & $\xi_{3}^{\prime}$ & $\xi_{4}^{\prime}$ \\
\hline$J_{x_{1}}$ & 1 & 2 & 2 & 1 & 1 & $1 / 2$ & 8 & 8 & 8 \\
$J_{\bar{x}_{1}}$ & 2 & 2 & 1 & 2 & 2 & $1 / 2$ & 8 & 8 & 8 \\
$J_{x_{2}}$ & 4 & 4 & 3 & 3 & 4 & 8 & $2+1 / 2$ & 8 & 8 \\
$J_{\bar{x}_{2}}$ & 3 & 3 & 4 & 4 & 4 & 8 & $2+1 / 2$ & 8 & 8 \\
$J_{x_{3}}$ & 6 & 6 & 6 & 5 & 5 & 8 & 8 & $4+1 / 2$ & 8 \\
$J_{\bar{x}_{3}}$ & 5 & 5 & 6 & 6 & 6 & 8 & 8 & $4+1 / 2$ & 8 \\
$J_{x_{4}}$ & 8 & 7 & 8 & 8 & 8 & 8 & 8 & 8 & $6+1 / 2$ \\
$J_{\bar{x}_{4}}$ & 8 & 8 & 7 & 8 & 7 & 8 & 8 & 8 & $6+1 / 2$ \\
\hline
\end{tabular}

NP-hard and not at all approximable even in the restrictive case, in which all job processing times and weights are equal to 1 . This negative result is true for each fixed $\alpha \in(0,1)$. It was shown in Kasperski and Zieliński (2016) that MIN-EXP $1\left|p_{j}=1\right| \max T_{j}$ is strongly NP-hard and hard to approximate within $7 / 6-\epsilon$ for any $\epsilon>0$. Hence, we immediately get from Theorem 1 that for each constant $\alpha \in[0,1)$, MiN-CVAR $\alpha 1\left|p_{j}=1\right| \max T_{j}$ is strongly NPhard and hard to approximate within $7 / 6-\epsilon$ for any $\epsilon>0$.

We consider now the problem with the total tardiness criterion. The deterministic $1 \| \sum T_{j}$ problem is known to be NP-hard (Lawler 1977). However, $1\left|p_{j}=1\right| \sum T_{j}$ is polynomially solvable [see, e.g., Brucker (2007)]. The following result characterizes the complexity of the minmax version of this problem:

Theorem 4 MIN-MAX $1\left|p_{j}=1\right| \sum T_{j}$ with uncertain due dates is strongly NP-hard and not approximable within $\frac{5}{4}-\epsilon$ for any $\epsilon>0$.

Proof We will show a reduction from the NP-complete 3SAT problem, in which we are given boolean variables $x_{1}, \ldots, x_{n}$, a collection of clauses $\mathcal{C}_{1}, \ldots \mathcal{C}_{m}$, where each clause is a disjunction of at most 3 literals (variables or their negations) and we ask if there is an assignment to the variables which satisfies all clauses [see, e.g., Garey and Johnson (1979)]. Given an instance of 3- SAT, we create two jobs $J_{x_{j}}$ and $J_{\bar{x}_{j}}$ for each variable $x_{j}, j \in[n],|J|=2 n$. A due date scenario $\xi_{i}$ corresponding to clause $\mathcal{C}_{i}=\left(l_{1} \vee l_{2} \vee l_{3}\right)$ is created in the same way as in the proof of Theorem 3. Additionally, for each variable $x_{j}$ we create scenario $\xi_{j}^{\prime}$ under which the due dates of $J_{x_{j}}$ and $J_{\bar{x}_{j}}$ are $2(j-1)+\frac{1}{2}$ and the due dates of the remaining jobs are set to $2 n$ (see Table 5). We first show that the answer to 3- SAT is yes if and only if there is a schedule $\pi$ such that $\max _{\xi \in \mathcal{U}} \sum_{j \in J} T_{j}(\pi, \xi) \leq 2$.

Assume that the answer to 3 -SAT is yes. Consider schedule $\pi=\left(J_{1}, J_{1}^{\prime}, J_{2}, J_{2}^{\prime}, \ldots, J_{n}, J_{n}^{\prime}\right)$, where $J_{j}, J_{j}^{\prime} \in$ $\left\{J_{x_{j}}, J_{\bar{x}_{j}}\right\}$. Furthermore, $J_{x_{j}}$ is processed before $J_{\bar{x}_{j}}$ if and only if $x_{j}=1$. Since in every clause at least one literal is true, at most two jobs in $\pi$ are late under each scenario $\xi_{i} \in \mathcal{U}$. The tardiness of each job in $\pi$ under any $\xi_{i} \in \mathcal{U}$ is at most 1 . Furthermore, the total tardiness in $\pi$ under any $\xi_{j}^{\prime}$ is exactly 2 . In consequence, $\max _{\xi \in \mathcal{U}} \sum_{j \in J} T_{j}(\pi, \xi) \leq 2$.

Assume that there is a schedule $\pi$, such that $\max _{\xi \in \mathcal{U}} \sum_{j \in J}$ $T_{j}(\pi, \xi) \leq 2$. We claim that $\pi=\left(J_{1}, J_{1}^{\prime}, J_{2}, J_{2}^{\prime}, \ldots, J_{n}, J_{n}^{\prime}\right)$, where $J_{j}, J_{j}^{\prime} \in\left\{J_{x_{j}}, J_{\bar{x}_{j}}\right\}$. Suppose that this is not the case, and let $J_{k}\left(J_{k}^{\prime}\right)$ be the last job in $\pi$ which is not placed properly. The completion time of $J_{k}\left(J_{k}^{\prime}\right)$ is at least $2 k+1$. So, its tardiness under $\xi_{k}^{\prime}$ is at least $2 k+1-\left(2 k-2+\frac{1}{2}\right)=2.5$. Let $x_{j}=1$ if and only if $J_{x_{j}}$ is processed before $J_{\bar{x}_{j}}$ in $\pi$. Since only two jobs can be late under any $\xi_{i}$, this assignment satisfies all clauses and the answer to 3- SAT is yes.

In order to prove the lower approximation bound, it is enough to observe that if the answer to 3-SAT is no, then each schedule has the total tardiness 3 under some scenario $\xi_{i}$ or 2.5 under some scenario $\xi_{j}^{\prime}$, which gives a gap at least $\frac{5}{4}$.

From the fact that $1 \| \sum T_{j}$ is weakly NP-hard [see Du and Leung (1990)], we get immediately that more general MIN-EXP $1 \| \sum T_{j}$ problem with uncertain due dates is weakly NP-hard as well. The next theorem strengthens this result.

Theorem 5 MIN-EXP $1 \| \sum T_{j}$ with uncertain due dates is strongly NP-hard.

Proof We will show a polynomial time reduction from the deterministic $1 \| \sum w_{j} T_{j}$ problem, which is known to be strongly NP-hard (Lawler 1977). Consider an instance of $1 \| \sum w_{j} T_{j}$. Let $W=\sum_{j \in J} w_{j}>0$ and $P=\sum_{j \in J} p_{j}$. We build an instance of MIN-EXP $1 \| \sum T_{j}$ with the same set of jobs $J$ and job processing times $p_{j}, j \in J$. We create $K=|J|=n$ due date scenarios as follows. Under scenario $\xi_{j}, j \in[n]$, job $j$ has due date equal to $d_{j}$ and all the remaining jobs have due dates equal to $P$. We also fix $\operatorname{Pr}\left[\xi_{i}\right]=w_{i} / W, i \in[n]$. For any schedule $\pi$, we get $\mathbf{E}[\mathrm{F}(\pi)]=\sum_{i \in[K]} \operatorname{Pr}\left[\xi_{i}\right] \sum_{j \in J} T_{j}\left(\pi, \xi_{i}\right)=$ $\frac{1}{W} \sum_{i \in[n]} w_{i} \sum_{j \in J} T_{j}\left(\pi, \xi_{i}\right)$. By the construction, we get $\sum_{j \in J} T_{j}\left(\pi, \xi_{i}\right)=\left[C_{i}(\pi)-d_{i}\right]^{+}$, so $\mathbf{E}[\mathrm{F}(\pi)]=\frac{1}{W} \sum_{i \in[n]}$ $w_{i}\left[C_{i}(\pi)-d_{i}\right]^{+}$. In consequence $1 \| \sum w_{j} T_{j}$ and MIN-EXP 1 ॥ $\sum T_{j}$ have the same optimal solutions and the theorem follows.

It was shown in Aissi et al. (2011) that MiN-MAX $1 \mid p_{j}=$ 1| $\sum U_{j}$ with uncertain due dates is strongly NP-hard. The following theorem strengthens this result:

Theorem 6 MIN-MAX $1\left|p_{j}=1\right| \sum U_{j}$ with uncertain due dates is not approximable within any constant factor unless $P=N P$. 
Proof Consider the following MIN-MAX 0-1 SELECTION problem. We are given a set of items $E=\left\{e_{1}, e_{2}, \ldots\right.$, $\left.e_{n}\right\}$ and an integer $q \in[n]$. For each item $e_{j}, j \in[n]$, there is a cost $c_{j}\left(\xi_{i}\right) \in\{0,1\}$ under scenario $\xi_{i}, i \in[K]$. We seek a selection $X \subseteq E$ of exactly $q$ items, $|X|=q$, which minimizes the maximum cost over all scenarios, i.e., the value of $\max _{i \in[K]} \sum_{e_{j} \in X} c_{j}\left(\xi_{i}\right)$. This problem was discussed in Kasperski et al. (2013), where it was shown that it is not approximable within any constant factor $\gamma>1$. We will show that there is a cost preserving reduction from MiNMAX 0-1 SELECTION to the considered scheduling problem, which will imply the stated result.

Given an instance of MIN-MAX 0-1 SELECTION, we build the corresponding instance of MIN-MAX $1\left|p_{j}=1\right| \sum U_{j}$ as follows. We create a set of jobs $J=E,|J|=n$, with deterministic unit processing times. For each $i \in[K]$, if $c_{j}\left(\xi_{i}\right)=1$ then $d_{j}\left(\xi_{i}^{\prime}\right)=n-q$, and if $c_{j}\left(\xi_{i}\right)=0$, then $d_{j}\left(\xi_{i}^{\prime}\right)=n$. So, we create $K$ due date scenarios that correspond to the cost scenarios of MIN-MAX 0-1 SELECTION.

Suppose that there is a solution $X$ to MIN-MAX 0-1 SELECTION such that $\sum_{e_{j} \in X} c_{j}\left(\xi_{i}\right) \leq C$ for each $i \in[K]$. Hence, $X$ contains at most $C$ items, $C \leq q$, with the cost equal to 1 under each scenario. In the corresponding schedule $\pi$, we first process $n-q$ jobs from $J \backslash X$ and then the jobs in $X$ in any order. It is easily seen that there are at most $C$ late jobs in $\pi$ under each scenario $\xi_{i}^{\prime}$, and hence, the maximum cost of schedule $\pi$ is at most $C$. Conversely, let $\pi$ be a schedule in which there are at most $C$ late jobs under each scenario $\xi_{i}^{\prime}$. Clearly $C \leq q$ since the first $n-q$ jobs in $\pi$ must be on-time in all scenarios. Let us form solution $X$ by choosing the items corresponding to the last $q$ jobs in $\pi$. Among these jobs at most $C$ are late under each scenario, and hence, the cost of $X$ is at most $C$ under each scenario $\xi_{i}$.

Thus, by Theorem 2, MIN-CVAR ${ }_{\alpha} 1\left|p_{j}=1\right| \sum U_{j}$ is strongly NP-hard for any $\alpha \in(0,1)$. (Notice that $p_{\max }=1$ in the proof of Theorem 2 and in the new scenario set $\mathcal{U}^{\prime}$ still only due dates are uncertain.)

\section{Theorem 7 MIN-EXP $1 \| \sum U_{j}$ with uncertain due dates is NP-hard.}

Proof Consider the deterministic $1 \| \sum w_{j} U_{j}$ problem, which is known to be NP-hard (Karp 1972). The reduction from this problem to MIN-EXP $1 \| \sum U_{j}$ is the same as the one in the proof of Theorem 5 .

It is worth noting that in the proof of Theorem 7 we require an arbitrary probability distribution in the scenario set and we have shown that the problem is only weakly NP-hard. Its complexity for uniform probability distribution is open.

\subsection{Uncertain processing times}

In this section, we characterize the complexity of the problems under consideration when only processing times are uncertain. It has been shown in Kouvelis and Yu (1997) that MIN-MAX $1 \| \sum C_{j}$ is strongly NP-hard. Furthermore, this problem is also hard to approximate within $\frac{6}{5}-\epsilon$ for any $\epsilon>0$ (Mastrolilli et al. 2013). Using Theorem 2, we can immediately conclude that the same negative result holds for MIN-VAR $\alpha 1 \| \sum C_{j}$ for any $\alpha \in(0,1]$. Also, strong NP-hardness of the min-max problem implies that MIN-CVAR ${ }_{\alpha} 1 \| \sum C_{j}$ is strongly NP-hard for each fixed $\alpha \in(0,1)$. Observe that the boundary case $\alpha=0$ (i.e., MIN-EXP $1 \| \sum C_{j}$ ) is polynomially solvable, as it easily reduces to the deterministic $1 \| \sum C_{j}$ problem. Since $1 \| \sum C_{j}$ is a special case of $1 \| \sum T_{j}$, with $d_{j}=0$ for each $j \in J$, the same negative results are true for the problem with the total tardiness criterion. Observe, however, that MIN-EXP $1 \| \sum T_{j}$ is also NP-hard, since the deterministic $1 \| \sum T_{j}$ problem is known to be weakly NP-hard (Du and Leung 1990).

It has been shown in Aloulou and Croce (2008) that MIN-MAX $1 \| \sum U_{j}$ with uncertain processing times and deterministic due dates is NP-hard. The following theorem strengthens this result:

Theorem 8 MIN-MAX $1 \| \sum U_{j}$ with uncertain processing times is strongly NP-hard. This assertion remains true even when all the jobs have a common deterministic due date.

Proof We show a polynomial time reduction from the 3-SAT problem (see the proof of Theorem 4). Given an instance of 3-SAT, we create an instance of MIN-MAX $1 \| \sum U_{j}$ in the following way. For each variable $x_{i}$, we create two jobs $J_{x_{i}}$ and $J_{\bar{x}_{i}}$, so $J$ contains $2 n$ jobs. The due dates of all these jobs are the same under each scenario and equal 2 . For each clause $C_{j}=\left(l_{1}, l_{2}, l_{3}\right)$, we construct processing time scenario $\xi_{i}$, under which the jobs $J_{\bar{l}_{1}}, J_{\bar{l}_{2}}, J_{\bar{l}_{3}}$ have processing time equal to 1 and all the remaining jobs have processing times equal to 0 . Then, for each pair of jobs $J_{x_{i}}, J_{\bar{x}_{i}}$ we construct scenario $\xi_{i}^{\prime}$ under which the processing times of $J_{x_{i}}, J_{\bar{x}_{i}}$ are 2 and all the remaining jobs have processing times equal to 0 . A sample reduction is shown in Table 6. We will show that the answer to 3-SAT is yes if and only if there is a schedule $\pi$ such that $\max _{\xi \in \mathcal{U}} \sum_{j \in J} U(\pi, \xi) \leq n$.

Assume that the answer to 3- SAT is yes. Then, there exists a truth assignment to the variables which satisfies all the clauses. Let us form schedule $\pi$ by processing first the jobs corresponding to true literals in any order and processing then the remaining jobs in any order. From the construction of the scenario set, it follows that the completion time of the $n$th job in $\pi$ under each scenario is not greater than 2 . In consequence, at most $n$ jobs in $\pi$ are late under each scenario and $\max _{\xi \in \mathcal{U}} \sum_{j \in J} U(\pi, \xi) \leq n$. 
Table 6 Processing time scenarios for the formula $\left(x_{1} \vee \bar{x}_{2} \vee \bar{x}_{3}\right) \wedge$ $\left(\bar{x}_{2} \vee \bar{x}_{3} \vee x_{4}\right) \wedge\left(\bar{x}_{1} \vee x_{2} \vee \bar{x}_{4}\right) \wedge\left(x_{1} \vee x_{2} \vee x_{3}\right) \wedge\left(x_{1} \vee x_{3} \vee \bar{x}_{4}\right)$. Schedule $\pi=\left(J_{x_{1}}, J_{\bar{x}_{2}}, J_{x_{3}}, J_{\bar{x}_{4}} \mid J_{\bar{x}_{1}}, J_{x_{2}}, J_{\bar{x}_{3}}, J_{x_{4}}\right)$ corresponds to a satisfying truth assignment

\begin{tabular}{lllllllllll}
\hline & $\xi_{1}$ & $\xi_{2}$ & $\xi_{3}$ & $\xi_{4}$ & $\xi_{5}$ & $\xi_{1}^{\prime}$ & $\xi_{2}^{\prime}$ & $\xi_{3}^{\prime}$ & $\xi_{4}^{\prime}$ & $d_{i}$ \\
\hline$J_{x_{1}}$ & 0 & 0 & 1 & 0 & 0 & 2 & 0 & 0 & 0 & 2 \\
$J_{\bar{x}_{1}}$ & 1 & 0 & 0 & 1 & 1 & 2 & 0 & 0 & 0 & 2 \\
$J_{x_{2}}$ & 1 & 1 & 0 & 0 & 0 & 0 & 2 & 0 & 0 & 2 \\
$J_{\bar{x}_{2}}$ & 0 & 0 & 1 & 1 & 0 & 0 & 2 & 0 & 0 & 2 \\
$J_{x_{3}}$ & 1 & 1 & 0 & 0 & 0 & 0 & 0 & 2 & 0 & 2 \\
$J_{\bar{x}_{3}}$ & 0 & 0 & 0 & 1 & 1 & 0 & 0 & 2 & 0 & 2 \\
$J_{x_{4}}$ & 0 & 0 & 1 & 0 & 1 & 0 & 0 & 0 & 2 & 2 \\
$J_{\bar{x}_{4}}$ & 0 & 1 & 0 & 0 & 0 & 0 & 0 & 0 & 2 & 2 \\
\hline & & & & & & & & & &
\end{tabular}

Assume that there is a schedule $\pi$ such that $\sum_{j \in J} U(\pi, \xi)$ $\leq n$ for each $\xi \in \mathcal{U}$, which means that at most $n$ jobs in $\pi$ are late under each scenario. Observe first that $J_{x_{i}}$ and $J_{\bar{x}_{i}}$ cannot appear among the first $n$ jobs in $\pi$ for any $i \in[n]$; otherwise, more than $n$ jobs would be late in $\pi$ under $\xi_{i}^{\prime}$. Hence, the first $n$ jobs in $\pi$ correspond to a truth assignment to the variables $x_{1}, \ldots, x_{n}$, i.e., when $J_{l}$ is among the first $n$ jobs, then the literal $l$ is true. Since $f\left(\pi, \xi_{i}\right) \leq n$, the completion time of the $n$-th job in $\pi$ under $\xi_{i}$ is not greater than 2 . We conclude that at most two jobs among the first $n$ job have processing time equal to 1 under $\xi_{i}$, so there are at most two false literals for each clause and the answer to 3-SAT is yes.

We thus get from Theorems 2 and 8 that MIN-VAR $\alpha 1 \|$ $\sum U_{j}$ is strongly NP-hard for any $\alpha \in(0,1]$ and MIN$\mathrm{CVAR}_{\alpha} 1 \| \sum U_{j}$ is strongly NP-hard for any $\alpha \in(0,1)$. The boundary case with $\alpha=0$ (i.e., MIN-EXP $1 \| \sum U_{j}$ with uncertain processing times) is an interesting open problem.

\section{Positive complexity results}

In this section we establish some positive complexity results. Namely, we provide several polynomial and approximation algorithms for particular problems. A summary of the results can be found in Table 3 .

\subsection{Problems with uncertain due dates}

Consider the MIN-EXP $1\left|p_{j}=1\right| \sum w_{j} U_{j}$ problem with uncertain due dates. We introduce variables $x_{i j} \in\{0,1\}$, $i \in[n], j \in[n]$, where $x_{i j}=1$ if $j \in[n]$ is the $i$ th job in the schedule constructed. The variables satisfy the assignment constraints, i.e., $\sum_{i \in[n]} x_{i j}=1$ for each $j \in[n]$ and $\sum_{j \in[n]} x_{i j}=1$ for each $i \in[n]$. If $x_{i j}=1$, then the completion time of job $j$ equals $i$. Define $c_{i j k}=w_{j}$ if $i>d_{j}\left(\xi_{k}\right)$ and $c_{i j k}=0$ otherwise, for each $i, j \in[n]$ and $k \in[K]$. If the variables $x_{i j}$ describe $\pi$, then
$\mathbf{E}[\mathrm{F}(\pi)]=\sum_{k \in[K]} \sum_{i \in[n]} \sum_{j \in[n]} \operatorname{Pr}\left[\xi_{k}\right] c_{i j k} x_{i j}=\sum_{i \in[n]} \sum_{j \in[n]} c_{i j}^{*} x_{i j}$,

where $c_{i j}^{*}=\sum_{k \in[K]} \operatorname{Pr}\left[\xi_{k}\right] c_{i j k}$. Hence, the problem is equivalent to the MINIMUM ASSIGNMENT with the cost matrix $c_{i j}^{*}$. The same result holds for MIN-EXP $1\left|p_{j}=1\right| \sum w_{j} T_{j}$. It is enough to define $c_{i j k}=w_{j}\left[i-d_{j}\left(\xi_{k}\right)\right]^{+}$for $i, j \in[n]$, $k \in[K]$. We thus get the following results:

Theorem 9 MIN-EXP $\mathcal{P}$ with uncertain due dates is polynomially solvable, when $\mathcal{P} \in\left\{1\left|p_{j}=1\right| \sum w_{j} U_{j}, 1 \mid p_{j}=\right.$ $\left.1 \mid \sum w_{j} T_{j}\right\}$

From Theorems 9 and 1, we immediately get the following approximation result:

Theorem $10 \mathrm{MIN}-\mathrm{CVAR}_{\alpha} \mathcal{P}$ with uncertain due dates is approximable within $\rho=\min \left\{\frac{1}{\operatorname{Pr}_{\min }}, \frac{1}{1-\alpha}\right\}$, when $\mathcal{P} \in$ $\left\{1\left|p_{j}=1\right| \sum w_{j} U_{j}, 1\left|p_{j}=1\right| \sum w_{j} T_{j}\right\}$.

Since MIN-MAX $1\left|p_{j}=1\right| \sum w_{j} U_{j}$ and MIN-MAX $1 \mid p_{j}$ $=1 \mid \sum w_{j} T_{j}$ are special cases of the min-max version of MiNIMUM ASSIGNMENT, which is approximable within $K$ [see, e.g., Aissi et al. (2009)], both problems are approximable within $K$ as well.

We now study the MIN-EXP $1 \| \sum w_{j} T_{j}$ problem with uncertain due dates and deterministic processing times. This problem is strongly NP-hard since $1 \| \sum w_{j} T_{j}$ is strongly NP-hard. The expected cost of $\pi$ can be rewritten as $\mathbf{E}[\mathrm{F}(\pi)]=\sum_{j \in J} \sum_{i \in[K]} \operatorname{Pr}\left[\xi_{i}\right]\left[C_{j}(\pi)-d_{j}\left(\xi_{i}\right)\right]^{+}$. We thus get a single machine scheduling problem $1 \| \sum f_{j}$ with job-dependent cost functions of form $f_{j}\left(C_{j}(\pi)\right)=$ $\sum_{i \in[K]} \operatorname{Pr}\left[\xi_{i}\right]\left[C_{j}(\pi)-d_{j}\left(\xi_{i}\right)\right]^{+}, j \in J$. Note also that these functions are nonnegative and nondecreasing with respect to $C_{j}(\pi)$. The same analysis can be done for the MIN-EXP $1 \| \sum w_{j} U_{j}$ problem with uncertain due dates and deterministic processing times. Hence and from Cheung et al. (2017), where a $(4+\epsilon)$-approximation algorithm, for any $\epsilon>0$, for this class of problems was provided, we get the following result (see also Theorem 1).

Theorem 11 If $\mathcal{P} \in\left\{1|| \sum w_{j} U_{j}, 1 \| \sum w_{j} T_{j}\right\}$, then MIN$\operatorname{ExP} \mathcal{P}$ with uncertain due dates is approximable within $4+\epsilon$ and $\mathrm{MIN}-\mathrm{CVAR}_{\alpha} \mathcal{P}$ is approximable within $\min \left\{\frac{4+\epsilon}{\operatorname{Pr}_{\min }}, \frac{4+\epsilon}{1-\alpha}\right\}$, $\epsilon>0$, for any $\epsilon>0$ and each constant $\alpha \in[0,1)$.

When the probability distribution in $\mathcal{U}$ is uniform, then the approximation ratio in Theorem 1 can be improved to $\min \left\{(4+\epsilon) K, \frac{4+\epsilon}{1-\alpha}\right\}$. Since MIN-MAX $\mathcal{P}$ is a special case of $\mathrm{MIN}-\mathrm{CVAR}_{\alpha} \mathcal{P}$ with uniform probability distribution and $\alpha$ sufficiently large, we get that MIN-MAX $\mathcal{P}$, $\mathcal{P} \in\left\{1\left\|\sum w_{j} U_{j}, 1\right\| \sum w_{j} T_{j}\right\}$, is approximable within $(4+\epsilon) K$ for any $\epsilon>0$. 


\subsection{The total weighted flow time criterion}

In this section, we focus on the problems with the total weighted flow time criterion. We start by recalling a wellknown property [see, e.g., Mastrolilli et al. (2013)], which states that every such a problem with uncertain processing times and deterministic weights can be transformed into an equivalent problem with uncertain weights and deterministic processing times. This transformation goes as follows. For each processing time scenario $\xi_{i}, i \in[K]$, we invert the role of processing times and weights obtaining the weight scenario $\xi_{i}^{\prime}$. Formally, $p_{j}=w_{j}$ and $w_{j}\left(\xi_{i}^{\prime}\right)=p_{j}\left(\xi_{i}\right)$ for each $i \in[K]$. The new scenario set $\mathcal{U}^{\prime}$ contains scenario $\xi_{i}^{\prime}$ with $\operatorname{Pr}\left[\xi_{i}^{\prime}\right]=\operatorname{Pr}\left[\xi_{i}\right]$ for each $i \in[K]$. We also invert the precedence constraints, i.e., if $i \rightarrow j$ in the original problem, then $j \rightarrow i$ in the new one. Given a feasible schedule $\pi=(\pi(1), \ldots, \pi(n))$, let $\pi^{\prime}=(\pi(n), \ldots, \pi(1))$ be the corresponding inverted schedule. Of course, schedule $\pi^{\prime}$ is feasible for the inverted precedence constraints. It is easy to verify that $f\left(\pi, \xi_{i}\right)=f\left(\pi^{\prime}, \xi_{i}^{\prime}\right)$ for each $i \in[K]$. In consequence $\mathbf{C V a R}_{\alpha}[\mathrm{F}(\pi)]=\mathbf{C V a r}_{\alpha}\left[\mathrm{F}^{\prime}\left(\pi^{\prime}\right)\right]$ and $\operatorname{VaR}_{\alpha}[\mathrm{F}(\pi)]=\operatorname{VaR}_{\alpha}\left[\mathrm{F}^{\prime}\left(\pi^{\prime}\right)\right]$, where $\mathrm{F}^{\prime}\left(\pi^{\prime}\right)$ is the random cost of $\pi^{\prime}$ for scenario set $\mathcal{U}^{\prime}$. Hence, the original problem with uncertain processing times and the new one with uncertain weights have the optimal solutions with the same performance measure.

From now on we make the assumption that the jobs have deterministic processing times $p_{j}, j \in J$ and $w_{j}\left(\xi_{i}\right)$ is the weight of job $j$ under scenario $\xi_{i}, i \in[K]$. The value of $\mathbf{C V a R}_{\alpha}[\mathrm{F}(\pi)]$, for a fixed schedule $\pi$, can be computed by solving the following optimization problem (see the formulation (1)b):

$$
\begin{array}{lll}
\min & \gamma+\frac{1}{1-\alpha} \sum_{i \in[K]} \operatorname{Pr}\left[\xi_{k}\right] u_{k} & \\
\text { s.t. } & \gamma+u_{k} \geq \sum_{j \in J} w_{j}\left(\xi_{k}\right) C_{j}(\pi) & k \in[K] \\
& u_{k} \geq 0 & k \in[K]
\end{array}
$$

Let $\delta_{i j} \in\{0,1\}, i, j \in[n]$, be binary variables such that $\delta_{i j}=1$ if job $i$ is processed before job $j$ in a schedule constructed. The vectors of all feasible job completion times $\left(C_{1}, \ldots, C_{n}\right)$ can be described by the following system of constraints (Potts 1980):

$$
\begin{array}{rlrl}
V C: & C_{j}=p_{j}+\sum_{i \in J \backslash\{j\}} \delta_{i j} p_{i} & j \in J \\
& \delta_{i j}+\delta_{j i}=1 & & i, j \in J, i \neq j \\
& \delta_{i j}+\delta_{j k}+\delta_{k i} \geq 1 & & i, j, k \in J \\
& \delta_{i j}=1 & & i \rightarrow j \\
& \delta_{i j} \in\{0,1\} & & i, j \in J
\end{array}
$$

Let us denote by $V C^{\prime}$ the relaxation of $V C$, in which the constraints $\delta_{i j} \in\{0,1\}$ are relaxed with $0 \leq \delta_{i j} \leq 1$. It has been proved in Schulz (1996) and Hall et al. (1997) that each vector $\left(C_{1}, \ldots, C_{n}\right)$ that satisfies $V C^{\prime}$ also satisfies the following inequalities:

$\sum_{j \in I} p_{j} C_{j} \geq \frac{1}{2}\left(\left(\sum_{j \in I} p_{j}\right)^{2}+\sum_{j \in I} p_{j}^{2}\right)$ for all $I \subseteq J$.

The formulations (7) and (6) lead to the following mixed integer programming model for MIN-CVAR $\alpha 1 \mid$ prec $\mid \sum w_{j} C_{j}$ with uncertain weights:

$$
\begin{array}{lll}
\min & \gamma+\frac{1}{1-\alpha} \sum_{i \in[K]} \operatorname{Pr}\left[\xi_{k}\right] u_{k} & \\
& & \\
\text { s.t. } & \gamma+u_{k} \geq \sum_{j \in J} w_{j}\left(\xi_{k}\right) C_{j} & k \in[K] \\
& \text { Constraints VC } & k \in[K] \\
& u_{k} \geq 0 &
\end{array}
$$

We now solve the relaxation of (9), in which $V C$ is replaced with $V C^{\prime}$. Let $\left(C_{1}^{*}, \ldots, C_{n}^{*}\right)$ be the relaxed optimal job completion times and $z^{*}$ be the optimal value of the relaxation. Consider discrete random variable $Y$, which takes the value $\sum_{j \in J} w_{j}\left(\xi_{i}\right) C_{j}^{*}$ with probability $\operatorname{Pr}\left[\xi_{i}\right], i \in[K]$. The equality $z^{*}=\mathbf{C V a R}_{\alpha}[Y]$ holds. We relabel the jobs so that $C_{1}^{*} \leq C_{2}^{*} \leq \cdots \leq C_{n}^{*}$ and form schedule $\pi=(1,2, \ldots, n)$ in nondecreasing order of $C_{j}^{*}$. Since the vector $\left(C_{j}^{*}\right)$ satisfies $V C^{\prime}$, it must also satisfy (8). Hence, setting $I=\{1, \ldots, j\}$, we get

$$
\sum_{i=1}^{j} p_{i} C_{i}^{*} \geq \frac{1}{2}\left(\left(\sum_{i=1}^{j} p_{i}\right)^{2}+\sum_{i=1}^{j} p_{i}^{2}\right) \geq \frac{1}{2}\left(\left(\sum_{i=1}^{j} p_{i}\right)^{2}\right) .
$$

Since $C_{j}^{*} \geq C_{i}^{*}$ for each $i \in\{1 \ldots j\}$, we get $C_{j}^{*} \sum_{i=1}^{j} p_{i} \geq$ $\sum_{i=1}^{j} p_{i} C_{i}^{*} \geq \frac{1}{2}\left(\sum_{i=1}^{j} p_{i}\right)^{2}$ and, finally $C_{j}=\sum_{i=1}^{j} p_{j} \leq$ $2 C_{j}^{*}$ for each $j \in J$-this reasoning is the same as in Schulz (1996).

For each scenario $\xi_{i} \in \mathcal{U}$, the inequality $f\left(\pi, \xi_{i}\right)=$ $\sum_{j \in J} w_{j}\left(\xi_{i}\right) C_{j} \leq 2 \sum_{j \in J} w_{j}\left(\xi_{i}\right) C_{j}^{*}$ holds. By Lemma 2, we have $\mathbf{C V a R}_{\alpha}[\mathrm{F}(\pi)] \leq 2 \cdot \mathbf{C V a R}_{\alpha}[Y]=2 z^{*}$. Since $z^{*}$ is a lower bound on the value of an optimal solution, $\pi$ is a 2-approximate schedule. Let us summarize the obtained result.

Theorem 12 MIN-CVAR ${ }_{\alpha} 1 \mid$ prec $\mid \sum w_{j} C_{j}$ with uncertain processing times is approximable within 2 for each $\alpha \in$ $[0,1)$.

This result can be refined when the deterministic $1 \mid$ prec $\mid \sum$ $w_{j} C_{j}$ problem is polynomially solvable [for example, when the precedence constraints form an sp-graph, see, e.g., 
Brucker (2007)]. In this case MIN-EXP $1 \mid$ prec $\mid \sum w_{j} C_{j}$ is polynomially solvable, and we can also apply Theorem 1 , which leads to the following result.

Theorem 13 If $1 \mid$ prec $\mid \sum w_{j} C_{j}$ is polynomially solvable, then $\mathrm{MIN}_{\mathrm{I}} \mathrm{CVAR}_{\alpha} 1 \mid$ prec $\mid \sum w_{j} C_{j}$ with uncertain processing times is approximable within $\min \left\{\frac{1}{1-\alpha}, 2\right\}$ for each $\alpha \in[0,1)$.

Observe that $\frac{1}{1-\alpha}<2$ for each $\alpha<0.5$. Let us consider MIN-VAR ${ }_{\alpha} 1 \mid$ prec $\mid \sum w_{j} C_{j}$ problem. The value of $\operatorname{VaR}_{\alpha}[\mathrm{F}(\pi)]$, for a fixed schedule $\pi$, can be computed by solving the following MIP problem (see (1)a):

$$
\begin{array}{ll}
\min & \theta \\
\text { s.t. } & \sum_{j \in J} w_{j}\left(\xi_{k}\right) C_{j}(\pi)-\theta \leq M_{k} \beta_{k} k \in[K] \\
& \sum_{\substack{k \in[K]\\
}} \operatorname{Pr}\left[\xi_{i}\right] \beta_{k} \leq 1-\alpha \\
& \beta_{k} \in\{0,1\}
\end{array}
$$

where $M_{k}$ is an upper bound on the schedule cost under scenario $\xi_{k}, k \in[K]$. Using the formulation (7) together with (1), we can get a mixed integer programming formulation for MIN-VAR ${ }_{\alpha} 1 \mid$ prec $\mid \sum w_{j} C_{j}$. By replacing the constraints $V C$ with relaxed $V C^{\prime}$ in the constructed model, we get a mixed integer problem with $K$ binary variables. This problem can be solved in polynomial time when $K$ is a constant. The same analysis as for MIN-CVAR ${ }_{\alpha} 1 \mid$ prec $\mid \sum w_{j} C_{j}$ (we also use Lemma 2) leads to the following result:

Theorem 14 If the number of scenarios is constant, then MIN-VAR ${ }_{\alpha} 1 \mid$ prec $\mid \sum w_{j} C_{j}$ with uncertain processing times is approximable within 2 for each $\alpha \in(0,1]$.

\subsection{The bottleneck objective}

In this section, we address a class of single machine scheduling problems with a bottleneck objective, i.e., in which $f(\pi)=\max _{j \in J} f_{j}\left(C_{j}(\pi)\right)$, where $f_{j}(t)$ is the cost of completing job $j$ at time $t$. An important and well-known example is $1 \mid$ prec $\mid \max w_{j} T_{j}$, in which the maximum weighted tardiness is minimized. This problem can be solved in $O\left(n^{2}\right)$ time by Lawler's algorithm (Lawler 1973). We will use the fact that the minmax versions of the bottleneck problems are polynomially solvable for a wide class of cost functions (Brauner et al. 2016; Kasperski and Zieliński 2016). In particular, the minmax version of $1 \mid$ prec $\mid \max w_{j} T_{j}$ with uncertain processing times and uncertain due dates can be solved in $O\left(K n^{2}\right)$ time by using the algorithm constructed in Kasperski and Zieliński (2016). In the following, we will assume that $f(\pi, \xi)=\max _{j \in J} w_{j} T_{j}(\pi, \xi)$ for a given scenario $\xi \in \mathcal{U}$. We also assume that job processing times and due dates are nonnegative integers under all scenarios and job weights are positive integers. In consequence, the value of $f(\pi, \xi)$ is a nonnegative integer for each $\xi$.

Let $f_{\max }$ be an upper bound on the schedule cost over all scenarios. Let $h: \mathbb{Q}_{+}^{K} \rightarrow \mathbb{Q}_{+}$be a nondecreasing function with respect to $\mathbb{Q}_{+}^{K}$, i.e., for any $\boldsymbol{t}, \boldsymbol{t}^{\prime} \in \mathbb{Q}_{+}^{K}$ if $t_{i}^{\prime} \leq t_{i}$ for each $i \in[K]$, then $h\left(t_{1}^{\prime}, \ldots, t_{K}^{\prime}\right) \leq h\left(t_{1}, \ldots, t_{K}\right)$. Suppose that $h$ can be evaluated in $g(K)$ time for a given vector $\boldsymbol{t}=\left(t_{1}, \ldots, t_{K}\right) \in \mathbb{Z}_{+}^{K}$. Consider the corresponding scheduling problem $\mathcal{P} \mathcal{S}$, in which we seek a feasible schedule $\pi \in \Pi$ minimizing $H(\pi)=h\left(f\left(\pi, \xi_{1}\right), \ldots, f\left(\pi, \xi_{K}\right)\right)$. We can find such a schedule by solving a number of the following auxiliary problems: given a vector $t \in \mathbb{Z}_{+}^{K}$, check if $\Pi(\boldsymbol{t})=\left\{\pi \in \Pi: f\left(\pi, \xi_{i}\right) \leq t_{i}, i \in[K]\right\}$ is nonempty, and if so, return any schedule $\pi_{t} \in \Pi(t)$. From the monotonicity of the function $h$, it follows that for each $\pi \in \Pi(t)$ the inequality $h\left(f\left(\pi, \xi_{1}\right), \ldots, f\left(\pi, \xi_{K}\right)\right) \leq h(\boldsymbol{t})$ is true. Thus, in order to solve the problem $\mathcal{P S}$, it suffices to enumerate all possible integral vectors $\boldsymbol{t}=\left(t_{1}, \ldots, t_{K}\right)$, where $t_{i} \in\left\{0,1, \ldots, f_{\max }\right\}, i \in[K]$, and compute $\pi_{\boldsymbol{t}} \in \Pi(\boldsymbol{t})$ if $\Pi(t)$ is nonempty. The number of such vectors is $\left(f_{\max }+1\right)^{K}$. A schedule $\pi_{t}$ with the minimum value of $H\left(\pi_{t}\right)$ is returned.

The crucial step in this method is solving the auxiliary problem. We now show that this can be done in polynomial time for the bottleneck problem with the maximum weighted tardiness criterion. Given any $t \in \mathbb{Z}_{+}^{K}$, we first form scenario set $\mathcal{U}^{\prime}$ by specifying the following parameters for each $\xi_{i} \in \mathcal{U}$ and $j \in J$ :

$$
\begin{aligned}
p_{j}\left(\xi_{i}^{\prime}\right) & =p_{j}\left(\xi_{i}\right), \quad w_{j}^{\prime}=1, \\
d_{j}\left(\xi_{i}^{\prime}\right) & =\max \left\{C \geq 0: w_{j}\left(C-d_{j}\left(\xi_{i}\right)\right) \leq t_{i}\right\} \\
& =t_{i} / w_{j}+d_{j}\left(\xi_{i}\right) .
\end{aligned}
$$

The scenario set $\mathcal{U}^{\prime}$ can be built in $O(K n)$ time. We then solve the minmax problem with scenario set $\mathcal{U}^{\prime}$, which can be done in $O\left(K n^{2}\right)$ time by using the algorithm constructed in Kasperski and Zieliński (2016). If the maximum cost of the schedule $\pi$ returned is 0 , then $\pi_{t}=\pi$; otherwise, $\Pi(t)$ is empty. Since all the risk criteria considered in this paper are nondecreasing functions with respect to schedule costs over scenarios (see Lemma 2 for $\gamma=1$ ) and $g(K)$ is negligible in comparison with $K n^{2}$, we get the following result.

Theorem 15 MIN-EXP $\mathcal{P}$, MIN-VAR $\alpha \mathcal{P}$ and $\mathrm{MIN}-\mathrm{CVAR}_{\alpha}$ $\mathcal{P}$ with uncertain processing times and uncertain due dates are solvable in $O\left(\left(f_{\max }+1\right)^{K} K n^{2}\right)$ time, when $\mathcal{P}$ is $1 \mid$ prec $\mid \max w_{j} T_{j}$.

The above running time is pseudopolynomial if $K$ is constant. Notice that the special cases, when $\mathcal{P}$ is $1 \mid$ prec,$p_{j}=$ 1| $\max T_{j}$ are solvable in $O\left(K(n+1)^{K+2}\right)$ time, which is polynomial if $K$ is constant (as we can fix $f_{\max }=n$ ).

We now show that the problems admit an FPTAS if $K$ is a constant and $h(\gamma \boldsymbol{t}) \leq \gamma h(\boldsymbol{t})$, for any $\boldsymbol{t} \in \mathbb{Q}_{+}^{K}, \gamma \geq 0$. 
First we partition the interval $\left[0, f_{\max }\right]$ into geometrically increasing subintervals: $[0,1) \cup \bigcup_{\ell \in[\eta]}\left[(1+\epsilon)^{\ell-1},(1+\epsilon)^{\ell}\right)$, where $\eta=\left\lceil\log _{1+\epsilon} f_{\max }\right\rceil$ and $\epsilon \in(0,1)$. Then, we enumerate all possible vectors $\boldsymbol{t}=\left(t_{1}, \ldots, t_{K}\right)$, where $t_{i} \in$ $\{0,1\} \cup \bigcup_{\ell \in[\eta]}\left\{(1+\epsilon)^{\ell}\right\}, i \in[K]$, and find $\pi_{t} \in \Pi(t)$ if $\Pi(t) \neq \emptyset$. Finally, we output a schedule $\pi_{\hat{t}}$ that minimizes value of $H\left(\pi_{t}\right)$ over the nonempty subsets of schedules. Obviously, the running time is $O\left(\left(\log _{1+\epsilon} f_{\max }\right)^{K}\left(K n^{2}+\right.\right.$ $g(K)))=O\left(\left(\epsilon^{-1} \log f_{\max }\right)^{K}\left(K n^{2}+g(K)\right)\right)$. Let $\pi^{*}$ be an optimal schedule. Fix $\ell_{i} \in\{0,1, \ldots, \eta\}$ for each $i \in[K]$, such that $(1+\epsilon)^{\ell_{i}-1} \leq f\left(\pi^{*}, \xi_{i}\right)<(1+\epsilon)^{\ell_{i}}$, where we assume that $(1+\epsilon)^{\ell_{i}-1}=0$ for $\ell_{i}=0$. This clearly forces $\Pi\left((1+\epsilon)^{\ell_{1}}, \ldots,(1+\epsilon)^{\ell_{K}}\right) \neq \emptyset$. Moreover, $(1+\epsilon)^{\ell_{i}} \leq$ $(1+\epsilon) f\left(\pi^{*}, \xi_{i}\right)$ for $\ell_{i}, i \in[K]$. By the definition of $\pi_{\hat{t}}$, we get $H\left(\pi_{\hat{\boldsymbol{t}}}\right) \leq h\left((1+\epsilon)^{\ell_{1}}, \ldots,(1+\epsilon)^{\ell_{K}}\right)$. Since $h$ is a nondecreasing function and $h(\gamma \boldsymbol{t}) \leq \gamma h(\boldsymbol{t}), h((1+$ $\left.\epsilon)^{\ell_{1}}, \ldots,(1+\epsilon)^{\ell_{K}}\right) \leq(1+\epsilon) h\left(f\left(\pi^{*}, \xi_{1}\right), \ldots, f\left(\pi^{*}, \xi_{K}\right)\right)$. Hence, $H\left(\pi_{\hat{t}}\right) \leq(1+\epsilon) H\left(\pi^{*}\right)$. By Lemma 2 , the risk criteria satisfy the additional assumption on the function $h(\boldsymbol{t})$. This leads to the following theorem:

Theorem 16 MiN-EXP $\mathcal{P}$, MiN-VAR $\alpha \mathcal{P}$ and $\mathrm{MIN}-\mathrm{CVAR}_{\alpha}$ $\mathcal{P}$ with uncertain processing times and uncertain due dates admit an FPTAS, when $\mathcal{P}$ is $1 \mid$ prec $\mid \max w_{j} T_{j}$ and the number of scenarios is constant.

It is worth pointing out that the problems from Theorem 16 are strongly NP-hard when the number of scenarios is a part of the input (see Sect. 5.1). If the number of scenarios is constant, then proving their weak NP-hardness is an interesting open problem.

\section{Conclusions and open problems}

In this paper, we have discussed a wide class of single machine scheduling problems with uncertain job processing times and due dates. This uncertainty is modeled by a discrete scenario set with a known probability distribution. In order to compute a solution, we have applied the risk criteria, namely the value at risk and conditional value at risk. The expectation and the maximum criteria are special cases of these risk measures. We have provided a number of negative and positive complexity results for problems with basic cost functions. Moreover, we have sharpened some negative ones obtained in Aissi et al. (2011) and Aloulou and Croce (2008). The picture of the complexity is presented in Tables 1,2 and 3 . Obviously, the negative results obtained remain true for more general cases, for instance, for the problems with more than one machine.

There is still a number of interesting open problems on the models discussed. The negative results for uncertain due dates assume that the number of due dates scenarios is a part of input. The complexity status of the problems when the number of due date scenarios is fixed (in particular, equals 2) is open. For uncertain processing times, an interesting open problem is MIN-EXP $1 \| \sum U_{j}$ (see Table 2). There is still a gap between the positive and negative results; in particular, we conjecture that the negative results for MIN-VAR $\mathcal{P}$ for uncertain processing times (see Table 2) can be strengthened. Now they are just the same as for the MIN-MAX $\mathcal{P}$.

Acknowledgements This work was supported by the National Science Centre, Poland, Grant 2017/25/B/ST6/00486.

Open Access This article is distributed under the terms of the Creative Commons Attribution 4.0 International License (http://creativecomm ons.org/licenses/by/4.0/), which permits unrestricted use, distribution, and reproduction in any medium, provided you give appropriate credit to the original author(s) and the source, provide a link to the Creative Commons license, and indicate if changes were made.

\section{References}

Aissi, H., Aloulou, M. A., \& Kovalyov, M. Y. (2011). Minimizing the number of late jobs on a single machine under due date uncertainty. Journal of Scheduling, 14, 351-360.

Aissi, H., Bazgan, C., \& Vanderpooten, D. (2009). Min-max and minmax regret versions of combinatorial optimization problems: A survey. European Journal of Operational Research, 197, 427-438.

Aloulou, M. A., \& Croce, F. D. (2008). Complexity of single machine scheduling problems under scenario-based uncertainty. Operations Research Letters, 36, 338-342.

Atakan, S., Bulbul, K., \& Noyan, N. (2017). Minimizng value-at-risk in single machine scheduling. Annals of Operations Research, 248, 25-73.

Averbakh, I. (2000). Minmax regret solutions for minimax optimization problems with uncertainty. Operations Research Letters, 27, 5765.

Avidor, A., \& Zwick, U. (2002). Approximating MIN k-SAT. Lecture Notes in Computer Science, 2518, 465-475.

Brauner, N., Finke, G., Shafransky, Y., \& Sledneu, D. (2016). Lawler's minmax cost algorithm: Optimality conditions and uncertainty. Journal of Scheduling, 19, 401-408.

Brucker, P. (2007). Scheduling algorithms (5th ed.). Heidelberg: Springer.

Chang, Z., Song, S., Zhang, Y., Ding, J.-Y., Zhang, R., \& Chiong, R. (2017). Distributionally robust single machine scheduling with risk aversion. European Journal of Operational Research, 256, 261274.

Cheung, M., Mestre, J., Shmoys, D. B., \& Verschae, J. (2017). A primal-dual approximation algorithm for min-sum single-machine scheduling problems. SIAM Journal on Discrete Mathematics, 31, 825-838.

Daniels, R. L., \& Kouvelis, P. (1995). Robust scheduling to hedge against processing time uncertainty in single-stage production. Management Science, 41, 363-376.

Drwal, M., \& Rischke, R. (2016). Complexity of interval minmax regret scheduling on parallel identical machines with total completion time criterion. Operations Research Letters, 44, 354-358.

Du, J., \& Leung, J. Y.-T. (1990). Minimizing total tardiness on one machine is NP-hard. Mathematics of Operations Research, 15, 483-495.

Garey, M. R., \& Johnson, D. S. (1979). Computers and intractability. A guide to the theory of NP-completeness. San Francisco: W. H. Freeman and Company. 
Hall, L. A., Schulz, A. S., Shmoys, D. B., \& Wein, J. (1997). Scheduling to minimize average completion time: Off-line and on-line approximation problems. Mathematics of Operations Research, $22,513-544$

Karp, R. M. (1972). Reducibility among combinatorial problems. In R. E. Miller \& J. W. Thatcher (Eds.), Complexity of computer computations (pp. 85-103). Plenum Press.

Kasperski, A. (2005). Minimizing maximal regret in the single machine sequencing problem with maximum lateness criterion. Operations Research Letters, 33(4), 431-436.

Kasperski, A., Kurpisz, A., \& Zieliński, P. (2013). Approximating the min-max (regret) selecting items problem. Information Processing Letters, 113, 23-29.

Kasperski, A., \& Zieliński, P. (2014). Minmax (regret) sequencing problems. In F. Werner \& Y. Sotskov (Eds.), Sequencing and scheduling with inaccurate data, chapter 8 (pp. 159-210). New York: Nova Science Publishers.

Kasperski, A., \& Zieliński, P. (2016). Single machine scheduling problems with uncertain parameters and the OWA criterion. Journal of Scheduling, 19, 177-190.

Kohli, R., Krishnamurti, R., \& Mirchandani, P. (1994). The minimum satisfiability problem. SIAM Journal on Discrete Mathematics, 7, 275-283.

Kouvelis, P., \& Yu, G. (1997). Robust discrete optimization and its applications. Dordrecht: Kluwer Academic Publishers.

Krokhmal, P., Palmquist, J., \& Uryasev, S. P. (2002). Portfolio optimization with conditional value-at-risk objective and constraints. Journal of Risk, 4, 43-68.

Lawler, E. L. (1973). Optimal sequencing of a single machine subject to precedence constraints. Management Science, 19, 544-546.

Lawler, E. L. (1977). A pseudopolynomial algorithm for sequencing jobs to minimize total tardiness. Annals of Discrete Mathematics, $1,331-342$.

Lebedev, V., \& Averbakh, I. (2006). Complexity of minimizing the total flow time with interval data and minmax regret criterion. Discrete Applied Mathematics, 154, 2167-2177.

Mastrolilli, M., Mutsanas, N., \& Svensson, O. (2013). Single machine scheduling with scenarios. Theoretical Computer Science, 477, $57-66$.

Möhring, R. H., Schulz, A. S., \& Uetz, M. (1999). Approximation in stochastic scheduling: The power of LP-based priority policies. Journal of the ACM, 46, 924-942.

Natarajan, K., Shi, D., \& Toh, K.-C. (2014). A probabilistic model for minmax regret in combinatorial optimization. Operations Research, 62, 160-181.
Nikolova, E. (2010). Approximation algorithms for offline risk-averse combinatorial optimization. In Proceedings of APPROX'10.

Ogryczak, W. (2012). Robust decisions under risk for imprecise probabilities. In Y. Ermoliev, M. Makowski, \& K. Marti (Eds.), Managing safety of heterogeneous systems (pp. 51-66). New York: Springer.

Pflug, G. C. (2000). Some remarks on the value-at-risk and the conditional value-at-risk. In S. P. Uryasev (Ed.), Probabilistic constrained optimization: Methodology and applications (pp. 272-281). Dordrecht: Kluwer Academic Publishers.

Pinedo, M. (2008). Scheduling: Theory, algorithms, and systems. New York: Springer.

Potts, C. N. (1980). An algorithm for the single machine sequencing problem with precedence constraints. Mathematical Programming Study, 13, 78-87.

Rockafellar, R. T., \& Uryasev, S. P. (2000). Optimization of conditional value-at-risk. The Journal of Risk, 2, 21-41.

Sarin, S., Sherali, H., \& Liao, L. (2014). Minimizing conditional-valueat-risk for stochastic scheduling problems. Journal of Scheduling, $17,5-15$.

Schulz, A. S. (1996). Scheduling to minimize total weighted completion time: Performance guarantees of LP-based heuristics and lower bounds. In IPCO (pp. 301-315).

Skutella, M., Sviridenko, M., \& Uetz, M. (2016). Unrelated machine scheduling with stochastic processing times. Mathematics of Operations Research, 41, 851-864.

Skutella, M., \& Uetz, M. (2005). Stochastic machine scheduling with precedence constraints. SIAM Journal on Computing, 34, 788 802.

Yager, R. R. (1988). On ordered weighted averaging aggregation operators in multi-criteria decision making. IEEE Transactions on Systems, Man and Cybernetics, 18, 183-190.

Yu, G., \& Kouvelis, P. (1993). Complexity results for a class of min-max problems with robust optimization applications. In P. M. Pardalos (Ed.), Complexity in numerical optimization. Singapore: World Scientific.

Publisher's Note Springer Nature remains neutral with regard to jurisdictional claims in published maps and institutional affiliations. 